\title{
Pots as alternative and sustainable fishing gears in the Mediterranean Sea: an overview
}

\author{
Andrea Petetta $(\mathbb{D} \cdot$ Massimo Virgili $\cdot$ Stefano Guicciardi $\cdot$ Alessandro Lucchetti
}

Received: 3 February 2021 / Accepted: 25 July 2021 / Published online: 9 August 2021

(C) The Author(s) 2021

\begin{abstract}
Stock overexploitation, bycatch, discards and gear impacts on the environment are outstanding issues for Mediterranean fisheries. The adoption of alternative fishing gears is an appealing solution to ensure a more sustainable exploitation of resources. We discuss the pros and cons of pots as alternative gears by reviewing their main designs, spatial distribution and target species in the Mediterranean basin. We assessed the technical factors affecting the catch efficiency of the different pot designs for four target species: spiny lobster, Palinurus elephas; Norway lobster, Nephrops norvegicus; common octopus, Octopus vulgaris and pandalid shrimps, Plesionika spp. We found that pot volume is important to catch Octopus; mesh size to catch Nephrops and Plesionika;
\end{abstract}

Andrea Petetta and Massimo Virgili have contributed equally to this work.

Supplementary Information The online version contains supplementary material available at https://doi.org/10.1007/ s11160-021-09676-6.

A. Petetta $(\bowtie)$

Department of Biological, Geological and Environmental Sciences (BIGEA), University of Bologna (UNIBO), Bologna, Italy

e-mail: andrea.petetta@irbim.cnr.it

A. Petetta - M. Virgili - S. Guicciardi - A. Lucchetti Institute for Biological Resources and Marine Biotechnologies (IRBIM), National Research Council (CNR), Ancona, Italy entrance surface to catch Octopus, Nephrops and Plesionika; pot shape/colour and entrance shape/position to catch Octopus and Plesionika; and bait type to catch Octopus and Nephrops. The literature review shows that pot fisheries have several considerable advantages over conventional gears, especially in terms of discards, bycatch, seabed impacts (particularly compared with bottom trawls and passive set nets), size and species selectivity, gear depredation, catch quality and gear cost, besides saving time and labour. Disadvantages hampering their wider diffusion include ghost fishing, a low catch of finfish species, the narrow range of species targeted by each pot design and the current early stage of research. These data make a clear case for using pots as alternative gears to traditional ones in the Mediterranean Sea in some areas and seasons to catch certain target species.

Keywords Sustainable fishery - Discard reduction . Alternative gear · Pots · Small-scale fishery . Mediterranean Sea

\section{Introduction}

Marine fisheries worldwide are faced with major sustainability issues at the ecosystem, economic and social levels (Jennings et al. 2016; Link and Watson 
2019) and the situation is hardly different for the Mediterranean. Overexploitation (Colloca et al. 2013) and the resulting fishing down the food webs (Coll et al. 2008), has been reported for more than $90 \%$ of the stocks assessed (Colloca et al. 2017; GFCM 2018). Bycatch also poses a significant threat, especially to large marine vertebrates such as sea turtles (Casale 2011; Lucchetti et al. 2017b, c), sharks (Ferretti et al. 2008; Bradai et al. 2018) and mammals (Bearzi 2002; Notarbartolo di Sciara 2016). In addition, high rates of discards, i.e. fish and other benthic organisms that are not retained for a variety of reasons-too small, damaged, inedible, of little or no commercial value, under the legal size or exceeding the allowed quotasare reported throughout the basin (Tsagarakis et al. 2014). Additional impacts involve physical alteration of the seabed (Lucchetti and Sala 2012; Palanques et al. 2014; Lucchetti et al. 2017a), disturbance of benthic habitats and communities (De Juan et al. 2007; Farriols et al. 2017) and greenhouse gas emissions (Guijarro et al. 2017), caused especially by towed gears.

The latest European Regulations aim to reduce all the impacts of fisheries with priority given to discard reduction. Until 2013, discarding in the Mediterranean was regulated by market demand rather than legal provisions, and marketable bycatch provided an important supplemental source of income (Tsagarakis et al. 2014). These practices eventually prompted the introduction of the Landing Obligation (LO) of all the catches of species subjected to catch quotas and, in the Mediterranean Sea, of those subject to the minimum conservation reference size (MCRS; EU Regulation, 1380/2013). In this basin, the LO aims at encouraging fishers to avoid areas or seasons characterized by large amounts of undersized / unwanted fish and to employ more selective gears. In recent years, the scientific community has been focusing trying to gain greater information on the spatial distribution of nursery areas of the main commercial species (Colloca et al. 2015) and has proposed spatiotemporal closures to minimize the catch of specimens under the MCRS (Russo et al. 2014; Despoti et al. 2020). The General Fisheries Commission for the Mediterranean (GFCM) has also established the Fisheries Restricted Areas (FRAs) to protect these essential fish habitats (FAO 2020). However, modifications to traditional fishing gear to improve size and species selectivity are among the most intensively investigated approaches. In bottom trawls such modifications usually involve the size, configuration, number and twine thickness of the codend meshes (Sala et al. 2007; Sala and Lucchetti 2011; Petetta et al. 2020a) or the use of bycatch reduction devices (BRDs) such as sorting grids and square mesh panels in the codend or the aft part of the extension piece (Bahamon et al. 2007; Tokaç et al. 2010; Brčić et al. 2017b; Lucchetti et al. 2019; Vasapollo et al. 2019; Bonanomi et al. 2020).

Passive nets are the most common gears used in small-scale fisheries (SSFs; Lucchetti et al. 2020). Several studies have evaluated the effect of different mesh sizes (Fabi et al. 2002; Karakulak and Erk 2008), netting twines (Ayaz et al. 2011; Grati et al. 2015) and hanging ratios (Kumova et al. 2015), whereas investigations assessing BRDs, such as the 'guarding net' mounted in trammel nets to reduce the unwanted catch of benthic invertebrates (Martínez-Baños and Maynou 2018; Sartor et al. 2018), and UV lights, applied to gill nets to reduce sea turtle bycatch (Virgili et al. 2018), are much less numerous.

Another solution to reduce discards, bycatch and seabed impacts is to adopt alternative fishing gear, both to ensure a more sustainable exploitation of resources (Petetta et al. 2020b) and to replace illegal gears (Notti et al. 2016). Recently, funding has been provided at the national and international levels through research projects (e.g., EMFF 2014-2020; BENTHIS; Plesionika Manage; TartaLife; Life Delfi), to investigate and promote pots as potential alternatives to traditional gears.

The current interest in pots (ICES 2007, 2008, 2009; Pol et al. 2010) is underpinned by their high potential to reduce the habitat impacts, fuel consumption, gear costs, discards and bycatch associated with passive nets and bottom trawls (Low Impact and Fuel Efficient, LIFE; Suuronen et al. 2012). Moreover, any discards removed from pots on board have considerable survival probability if they are not too stressed by factors such as barotrauma, air exposure and thermal shock (Suuronen et al. 2012). Notably, pots also provide greater catch quality (Olsen 2014) and are less subject to depredation (e.g. from dolphins and seals; Königson 2011; Pusch 2011) than passive nets. The research work conducted in the Mediterranean to test experimental pots has yielded good results in terms of catch efficiency, although further work is needed to improve their economic viability (Addis et al. 1998; Colloca 2002; Sartor et al. 
2006; Sbrana et al. 2008; Morello et al. 2009; Amengual-Ramis et al. 2016; Petetta et al. 2020b). Currently, the weight and value of landings generated by Mediterranean pot fisheries account for approximately 3800 tonnes and EUR 25.5 million, representing respectively $0.8 \%$ of fisheries landings and $1.5 \%$ of their revenues (STECF 2020). This study was undertaken to examine the commercial and experimental pot types employed in the FAO-GFCM geographical sub-areas (GSAs) of the Mediterranean. Its main objectives are (1) to provide an overview of the main pot designs, their spatial distribution and their main target species; (2) to assess the main factors affecting the catch efficiency of the different pot designs and gain insight into their retention mechanisms; and (3) to evaluate the main pros and cons of pots as alternatives to traditional fishing gears.

\section{Materials and methods}

\section{Pot characterization}

Pots are among the most traditional and diverse fishing gear employed in Mediterranean SSFs (Farrugio 2013), in line with the notion that primitive trapping is probably the oldest form of fishing (Slack-Smith 2001). In the FAO fishing gear catalogue (ISSCFG 2016), a pot is classified as a trap subtype (FPO08.2).

Briefly, pots are passive gear that attract and retain fish, crustaceans and molluscs with bait or pasture. They are small enclosures with one or more entrances which allow easy entry but make exit difficult (Pol et al. 2010). Bait is not requited when targeting species that seek a shelter or a hollow space in which to lay eggs, like cephalopods (Sobrino et al. 2011). Pots come in several different shapes and commonly consist of a rigid or semi-rigid frame in natural or artificial materials. The entrance(s) may have funnel(s), usually with a larger external opening that allows access to one or more inner chambers and a narrower internal opening preventing escape. Pots may be set on the bottom or allowed to float, according to the species being targeted, and may be single or stringed along branch lines that connect to a mainline (longline system). They are movable gears that after a variable soak time (hours to days) are hauled on deck, by hand or by mechanized haulers, and emptied. These features set pots aside from other traps such as fyke nets (FYK-08.3), which are left in the same place for longer periods and only their final parts or chambers are periodically hauled to collect the catch. Only gear falling in the definition of pots are reviewed herein.

\section{Data collection}

The existing pot designs were identified from material published in English, Spanish, French and Italian in peer-reviewed journals and in the grey literature, e.g., research project, reports and conference proceedings, using Google Search ${ }^{\odot}$ and Google Scholar ${ }^{\odot}$. Commercial pots employed in fisheries and experimental pots tested in scientific studies were both included, as was literature on brackish waters and lagoon environments.

For a reference to be selected, it had to provide at least information on GSA and target species. The technical data collected were grouped into three macro-categories: pot structure, netting mesh (if present) and entrance. In turn, pot structure was divided into shape, colour, frame and volume; netting mesh was divided into material, type and size; and entrance was divided into number, shape, position and surface. Each macro-category included one quantitative parameter (volume for pot structure, size for mesh and surface for entrance), whereas the remaining parameters were qualitative. Details about fishing depth and bait, if used, were also recorded.

\section{Data standardization}

The data collected for each parameter were subdivided into the smallest possible number of groups.

Depth range The depth of pot deployment was divided into four ranges: $<50,50-100,100-500$ and $>500 \mathrm{~m}$.

Pot shape The different pot shapes were grouped into six classes: clay, conical, cylindrical, parallelepiped, spherical and other. 'Clay' included all vase-shaped pots made of clay; 'conical' included conical, semi-conical, truncated-conical and bellshaped pots; 'cylindrical' included cylindrical, semicylindrical and tube-shaped pots; 'parallelepiped' included parallelepiped-shaped and rectangular boxshaped pots; 'spherical' included spherical and semispherical pots; 'other' included all other types, like arrow-shaped, pentagonal, ellipsoidal, semi- 
ellipsoidal, triangular-based prism and other irregular shapes.

Pot colour The external colour of the pot or the colour of the netting, if present.

Pot frame Pot frames were grouped into four types based on material: clay (clay, terracotta, ceramic), metal (aluminium, steel, iron, galvanized iron, galvanized tin, metal-covered wood, plastic-covered iron), natural (wood, rushes, intertwined reeds), and plastic (polyvinyl chloride [PVC], PVC-covered wood, generic plastic).

Pot volume This parameter was calculated from the pot dimensions reported in the references (length, height, width) and was expressed in litres (L). Designs with irregular shapes, which did not allow calculating volume using standard geometric formulae, were approximated to the closest regular shapes.

Mesh material. These were grouped into six types: polyamide (PA), polyethylene (PE), polypropylene (PP), natural (rushes, reeds), metal (steel or iron wire netting) and plastic (unspecified plastic material).

Mesh type Meshes were divided by shape into diamond, square, hexagonal, triangular and rectangular.

Mesh size. This parameter was intended as stretched mesh length, i.e., the distance between the centre of two opposite knots in the same mesh when fully extended in $\mathrm{N}$-direction, expressed in millimetres $(\mathrm{mm})$; the other mesh measurements were converted to this parameter.

Number of entrances One, two, or more entrances.

Entrance shape Entrance shape was grouped into six types: round with/without funnel, oval with/ without funnel, and rectangular with/without funnel.

Entrance position This parameter was classified in relation to the number of entrances. In pots with a single entrance, position was described as being on the short, long or upper side. In pots with two or more entrances, their position and alignment (opposite, not opposite) was recorded.

Entrance surface Surface was calculated from the entrance dimensions reported in the references (diameter, radius or side length) using standard geometric formulas and expressed in square centimetres $\left(\mathrm{cm}^{2}\right)$. In the types with funnel(s), the surface considered was that of the internal opening.

Bait Bait was grouped into six types: crabs, fish, molluscs, mixed (more than one type), other and no bait.
Statistical analysis

The catch abundance (number of individuals) and/or biomass (grams) data, reported in the references for the main target species, were subjected to statistical analysis to determine the catch performance related to each pot design and technical parameter. Tests were performed using data from three or more references per species.

Data from single hauls (where possible) or mean catch values were used for the analyses. The data were then standardized as number of individuals per pot per year ( $\mathrm{N}$ ind $\operatorname{pot}^{-1} \mathrm{year}^{-1}$ ) to calculate the catch abundance index (CAI), and as grams per pot per year $\left(\mathrm{g} \mathrm{pot}^{-1}\right.$ year $\left.^{-1}\right)$ to calculate the catch biomass index (CBI). The year time unit allowed obtaining integer values of the response variables. A quasi-Poisson was considered as the distribution family since the variance often differed from the mean. The catch indices were used as dependent variables to identify any significant relationships with the covariates (independent variables).

A generalized linear model (GLM) regression was performed for the three numerical covariates (pot volume, mesh size and entrance surface). These covariates showed mutual albeit not very strong correlations, as the maximum Pearson correlation coefficient was between pot volume and entrance surface (0.28). Since this could affect the conclusions of a multivariate GLM regression (Agresti 2015), a univariate GLM regression was performed to isolate the effect of each covariate and determine its strength and direction with respect to the response variable. For each target species and covariate, the significance of the relationship was evaluated based on the $p$ value; a $p$ value $<0.05$ indicated a significant effect of the covariate on the dependent variable.

The Kruskal-Wallis $\mathrm{H}$ test (which follows a $\chi^{2}$ distribution with n-1 degree of freedom; Witte and Witte 2017) was applied in case of factorial covariates (pot shape, pot colour, entrance shape, entrance position, bait type). A $p$ value of 0.05 was again considered as the significance threshold. In case of a significant $p$ value, Dunn's post-hoc test was performed to compare the different levels of the factors and to identify which level was significantly higher than the other ones. 
The free statistical software R (R Core Team 2019), and the FSA package (ver. 0.8.30) for the post-hoc test, were used for the analyses.

\section{SWOT analysis}

A SWOT analysis (Dyson 2004; Dana 2012) was performed to identify the Strengths and Weaknesses of the pot fisheries investigated in the review and the Opportunities and Threats related to using pots instead of traditional gear in the Mediterranean. The Strengths and Weaknesses were internal factors such as cost, facilities, fishery impacts and quality of products; the Opportunities and Threats were external factors relating to the economic, social, technological and sustainability levels of the fishery.

The search for key factors was performed by assessing comparative studies (pots vs other gears), including ones conducted in areas outside the Mediterranean, investigating the same target species as the present work. A factor was considered as a key factor if it was investigated at least three times in the pool of references.

Key factors for the analysis were evaluated based on results and suggestions obtained from the studies selected for the review. A 3-point score was assigned to each key factor as follows: 0 , no effect; 1 , medium effect; and 2, strong effect. References discussing at least five key factors were included in the SWOT analysis. A final ranking of the mean scores of each key factor was made to sketch the resulting scenario.

\section{Results}

Photographs of the main pot designs used in the Mediterranean to catch the target species examined in this work are provided as supplementary material (Suppl. Mat. 1).

\section{Technical data}

A total number of 107 references concerning the pots deployed in the Mediterranean Sea were reviewed. They covered more than 50 years (1968-2021) and 24 GSAs. The main species targeted by pots in each GSA are listed in Fig. 1. There were 15 species and 2 genera. In addition, three classes, 'shrimp', 'fish' and 'crab', were created to group references with unspecified target species or with more than one main target species. Common octopus, Octopus vulgaris was the species targeted in most GSAs $(n=16)$, followed by fish $(\mathrm{n}=11)$, spiny lobster, Palinurus elephas $(\mathrm{n}=9)$ and pandalid shrimps of the genus Plesionika $(\mathrm{n}=9)$. The GSAs with the highest number of species $(\mathrm{n}=8)$ targeted by pots were GSAs 6,17 and 22. Fishing depth and the technical characteristics of pots are reported according to target species in Table 1. A detailed description with references is given in the Appendix to Table 1 (Suppl. Mat. 2).

Depth range Most pots are deployed at $<50 \mathrm{~m}$. A small number of species-all crustaceans (deep-water red shrimp, Aristeus antennatus; European lobster, Homarus gammarus, Norway lobster, Nephrops norvegicus, pandalid shrimps and the 'shrimp' class-are exclusively caught in deeper waters (50-1500 m).

Pot shape It varies significantly in relation to target species and the same species is often targeted by different pot designs (spiny lobster, common octopus, pandalid shrimps). In a few cases, a single pot design is recorded for some species: groupers, Ephinephelus spp., GSA 24; sparids, GSAs 24 and 27; mantis shrimp, Squilla mantis, GSA 17; changeable nassa, Nassarius mutabilis, GSAs 6 and 17; and the banded dye murex, Hexaplex trunculus, GSA 22.

Pot volume Pots also come in a variety of volumes, even to target the same species. The smallest pots (1.6 L) are the gargoulettes employed for common octopus in the Gulf of Gabès (GSA 14), the largest (444 L) are the bell-shaped pots traditionally employed to catch the black seabream, Spondyliosoma cantharus, the Mediterranean moray, Muraena helena and other fish in Sardinia (GSA 11). The pots designed to target Norway lobster have a more limited variability, mostly ranging from 51 to $79 \mathrm{~L}$ (GSA 17).

Pot colour Most commercial pots are black or white. Green is also widely used, especially to target spiny lobster, pandalid shrimps, fish and cuttlefish, Sepia officinalis Brown is the typical colour of clay and reed pots.

Pot frame and mesh material. The two elements are closely related. Metal frames (mostly steel and iron) covered with PA or wire netting are the most common. Plastic pots are mainly used to catch common octopus. Frames and netting in natural materials, mostly intertwined reeds, are rare and are almost exclusively associated with bell-shaped pots (European conger, 


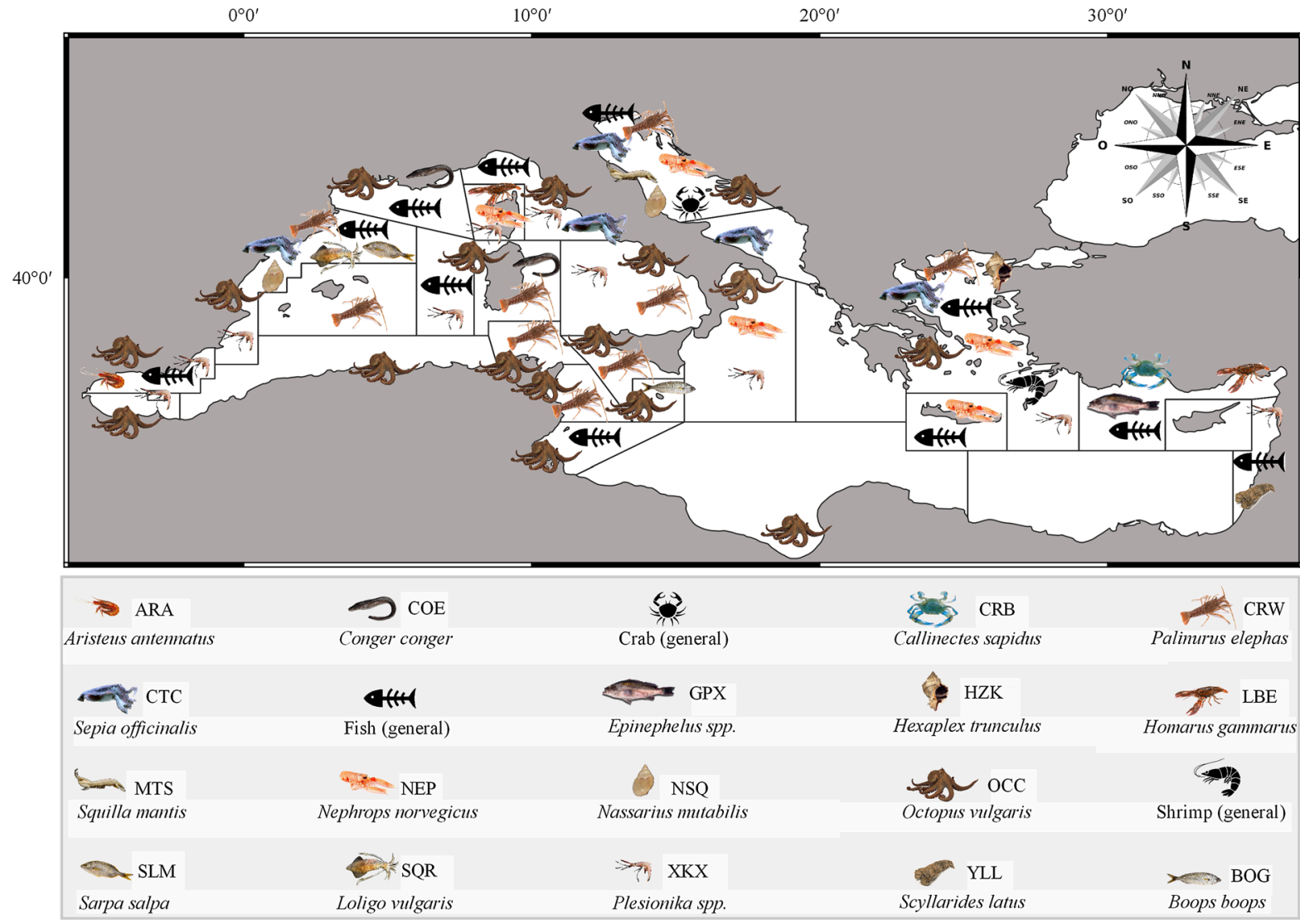

Fig. 1 Main species targeted with pots in each geographical sub-area (GSA) of the General Fisheries Commission for the Mediterranean

Conger conger and other fish, GSAs 11 and 14; spiny lobster, GSAs 5, 6, 10, and 16).

Mesh type and size The square mesh is more frequent than the other configurations (hexagonal, diamond, triangular). Mesh size varies widely among and within species.

Entrance number and position Pots with a single entrance are predominant (e.g., clay pots for common octopus and spherical pots for pandalid shrimps). In pots with two entrances, they may be set in opposite position (e.g., parallelepiped pots targeting cuttlefish or blue crab) or not in opposite position (e.g., the cylindrical Scottish design employed for Norway lobster). Three entrances are recorded only in a pentagonal pot deployed on a commercial scale in Croatia to target fish, cuttlefish and octopus.

Entrance shape It is commonly oval or round, rarely rectangular and never square. A funnel is usual, except in models where the entrance is on the upper side, which causes the specimen to fall into the pot. A funnel is not needed in clay pots deployed to attract common octopus.

Entrance surface This feature is also highly variable. The smallest entrances, ranging from 47 to 76 $\mathrm{cm}^{2}$, are found in pots targeting Norway lobster. The pots targeting spiny lobster in the Aegean Sea (GSA 22) have the largest entrances $\left(1256 \mathrm{~cm}^{2}\right)$.

Bait Fish and mixed bait are the main types. Fishused fresh, frozen, salted or fermented-mostly includes species of modest economic value. Bivalves are rarely used as bait. Crabs are used dead (e.g., to target Nephrops in GSA 17) or alive (e.g., to catch common octopus in GSA 9). Bait is not used to catch cuttlefish, which is attracted by laurel leaves, plastic ribbons and other substrates suitable for laying eggs. 
Table 1 Fishing depth and technical characteristics of the pots used in the Mediterranean Sea, listed by target species

\begin{tabular}{|c|c|c|c|c|c|c|c|c|c|c|c|c|c|}
\hline \multirow[b]{2}{*}{ Species } & \multirow[b]{2}{*}{ Depth } & \multicolumn{4}{|c|}{ Pot structure } & \multicolumn{3}{|c|}{ Netting mesh } & \multicolumn{4}{|c|}{ Entrance } & \multirow[b]{2}{*}{ BAIT } \\
\hline & & Shape & Colour & Frame & Vol (L) & Material & Type & $\begin{array}{c}\text { Size } \\
(\mathrm{mm})\end{array}$ & Number & Shape & Position & $\begin{array}{c}\text { Surface } \\
\left(\mathrm{cm}^{2}\right)\end{array}$ & \\
\hline ARA & $\varnothing$ & - & - & - & - & & - & - & - & - & - & - & - \\
\hline BOG & $\varnothing$ & O & - & $\mathrm{N}$ & - & - & - & - & - & - & - & - & - \\
\hline $\mathrm{COE}$ & $\varnothing$ & $\Delta$ & $\sigma$ & $\mathrm{N}$ & $141-445$ & $\mathrm{~N}$ & $\Delta$ & - & 1 & $\mathrm{CF}$ & SS & - & MIX MO \\
\hline Crab & $\varnothing$ & - & - & - & - & - & - & - & - & - & - & - & - \\
\hline CRB & $\varnothing$ & in & $\infty$ & $\mathrm{M}$ & $68-175$ & PA & $\square$ & $13-100$ & 2 & CF RF & $\mathrm{LS}(\mathrm{O}) \mathrm{SS}(\mathrm{O})$ & $66-452$ & FH NO \\
\hline CRW & $\varnothing \varnothing$ & ADI & $\square \square$ & $\begin{array}{c}\text { M N } \\
P\end{array}$ & $60-445$ & $\begin{array}{c}\text { M N PA PE } \\
\text { P }\end{array}$ & $\Delta \square$ & $15-80$ & $1-2$ & $\mathrm{CF} \mathrm{OF}$ & $\begin{array}{c}\text { SS SS(O) } \\
\text { US }\end{array}$ & $227-1256$ & FH \\
\hline CTC & $\varnothing$ & $\Delta=$ & $\bullet 0$ & M & $101-268$ & PA PE P & $\square$ & $22-56$ & $1-2$ & CF RF & $\begin{array}{l}\text { SS LS(O) } \\
\text { SS (Ø) }\end{array}$ & $154-565$ & NO \\
\hline Fish & $\varnothing \varnothing$ & $\Delta \|$ & 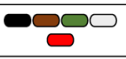 & $\mathrm{M} \mathrm{N}$ & $33-1800$ & $\begin{array}{c}\text { M N PA PE } \\
\text { P }\end{array}$ & $\diamond \square \square \Delta$ & $10-70$ & $1-3$ & $\begin{array}{c}\text { CF OF } \\
\text { RF }\end{array}$ & $\begin{array}{c}\text { LS SS } \\
\text { SS(O) US }\end{array}$ & $28-565$ & $\begin{array}{l}\text { FH MIX } \\
\text { OTH NO }\end{array}$ \\
\hline HZK & $\varnothing$ & 든. & 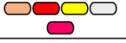 & $\mathrm{P}$ & $8-24$ & - & - & - & 1 & $\mathrm{C}$ & US & 79 & FISH \\
\hline GPX & $\varnothing$ & 0 & $\varnothing$ & $\mathrm{M}$ & $59-166$ & $\mathrm{M}$ & $\Delta$ & - & 1 & $\mathrm{CF}$ & US & 113 & $\mathrm{FH}$ \\
\hline LBE & $\square$ & an & $\sigma$ & $\mathrm{M}$ & 79-393 & $\mathrm{M}$ & $\square$ & 30 & 1 & CF OF & SS US & 491 & $\mathrm{FH}$ \\
\hline MTS & $\square$ & U & $\square \varnothing$ & M & 7 & $\mathrm{M}$ & $\square$ & 20 & 1 & OF & SS & 50 & $\mathrm{FH}$ \\
\hline NEP & $\square \varnothing$ & 10 & $\infty \square \square \square$ & $\mathrm{M}$ & $51-200$ & M PA P & $\diamond \square$ & $12-44$ & $1-2$ & $\mathrm{CF} \mathrm{OF}$ & $\mathrm{LS}(\varnothing) \mathrm{SS}(\mathrm{O})$ & $47-76$ & FH MIX CR \\
\hline NSQ & $\square$ & $\Delta$ & $\varnothing \square$ & $\mathrm{M}$ & $11-15$ & PA & $\square$ & $18-28$ & 1 & $\mathrm{C}$ & SS US & $177-314$ & FH MIX \\
\hline OCC & $\square$ & 814 & 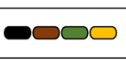 & $\begin{array}{c}\mathrm{C} \mathrm{M} \\
\mathrm{P} \\
\end{array}$ & $2-42$ & PA & $\square$ & - & $1-2$ & $\mathrm{CF} \mathrm{C}$ & $\begin{array}{c}\mathrm{SS} \mathrm{SS}(\mathrm{O}) \\
\mathrm{US}\end{array}$ & $79-314$ & $\begin{array}{c}\text { CR F MIX } \\
\text { NO }\end{array}$ \\
\hline Shrimp & $\theta$ & 0 & $\varnothing \oslash$ & $\mathrm{M}$ & 54 & PA PP & $\square \square$ & $14-40$ & 1 & OF & US & $314-1256$ & $\mathrm{FH}$ \\
\hline SLM & $\square$ & O & $\square \emptyset$ & $\mathrm{NP}$ & 90 & N P & $\square \Delta$ & - & 1 & $\mathrm{CF}$ & US & $113-177$ & OTH \\
\hline SQR & - & - & - & - & - & & - & - & - & - & - & - & - \\
\hline $\mathrm{XKX}$ & $\square \emptyset$ & $\Delta \square$ & $\sqsupset \square \square$ & M P & 53-385 & M P PP & $\square \diamond \square \square$ & $16-50$ & $1-2$ & CF OF C & $\begin{array}{c}\text { SS SS(O) } \\
\text { US }\end{array}$ & $50-133$ & FH MIX \\
\hline YLL & $\varnothing$ & $\Delta$ & - & $\mathrm{M}$ & - & - & - & - & - & - & US & - & MO \\
\hline
\end{tabular}

Target species are described by 3-alpha FAO Code. Further details are available in the Appendix to the table (Suppl. Mat. 2)

Depth range: $\square=<50 \mathrm{~m} ; \square=50-100 \mathrm{~m} ; \square=100-500 \mathrm{~m} ; \square=>500 \mathrm{~m}$

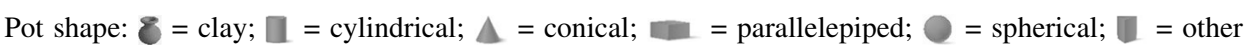

Pot colour: $\square$ =black; $\square$ = brown; $\square$ = green; $\square$ = grey; $\square$ = orange; $\square$ = pink; $\square=$ red, $\square=$ white; $\square=$ yellow; $\square=$ magenta

Pot frame: $\mathrm{C}=$ clay; $\mathrm{M}=$ metal; $\mathrm{N}=$ natural material; $\mathrm{P}=$ plastic

Mesh material: $\mathrm{N}=$ natural; $\mathrm{M}=$ metal; $\mathrm{PA}=$ polyamide; $\mathrm{PE}=$ polyethylene; $\mathrm{PP}=$ polypropylene; $\mathrm{P}=$ plastic $(\mathrm{generic})$

Mesh type: $\diamond=$ diamond; $\square=$ hexagonal; $\square=$ square; $\Delta=$ triangular

Entrance shape: $\mathrm{CF}=$ round with funnel; $\mathrm{C}=$ round without funnel; $\mathrm{OF}=$ oval with funnel; $\mathrm{RF}=$ rectangular with funnel

Entrance position: LS = long side; SS = short side; US = upper side; LS $(\mathrm{O})=$ long side, opposite; $\operatorname{LS}(\varnothing)=1$ long side, not opposite; $\mathrm{SS}(\mathrm{O})=$ short side, opposite

Bait type: $\mathrm{CR}=$ crabs; $\mathrm{FH}=$ fish; $\mathrm{MO}=$ mollusc; $\mathrm{MIX}=$ mixed; $\mathrm{NO}=$ no bait; $\mathrm{OTH}=$ other

Statistical analysis

Statistical analysis was performed on data from the four species for which more than three references reporting catch data were available: spiny lobster, Norway lobster, common octopus and pandalid shrimps. The results are reported below for each target species and are summarized in Table 2 (GLM results) and Table 3 (Kruskal-Wallis and Dunn's post- hoc test). The significant relationships identified by the GLM are represented graphically in Fig. 2.

\section{Spiny lobster}

CAI showed no significant relationship with the three numerical covariates (pot volume, mesh size, entrance surface), whereas no data were available for CBI. No significant effects were found between the catch data 
Table 2 Results of the generalized linear model (GLM) where CAI (N.ind $\cdot \operatorname{pot}^{-1} \cdot \mathrm{yr}^{-1}$ ) and CBI $\left(\mathrm{g} \cdot \mathrm{pot}^{-1} \cdot \mathrm{yr}^{-1}\right) \operatorname{are~} \mathrm{response}$ variables and pot volume, mesh size and entrance surface are numerical covariates for each species analysed

\begin{tabular}{|c|c|c|c|c|c|c|}
\hline Species & Catch index & Covariate & Estimate & Std. error & $t$ value & $p$ value \\
\hline \multirow[t]{6}{*}{ Spiny lobster } & \multirow[t]{6}{*}{ CAI } & Intercept & 5.773142 & 0.852469 & 6.772 & 0.007 \\
\hline & & Pot volume & -0.003628 & 0.005063 & -0.717 & 0.525 \\
\hline & & Intercept & -3.32632 & 3.72281 & -0.893 & 0.437 \\
\hline & & Mesh size & 0.22511 & 0.09357 & 2.406 & 0.095 \\
\hline & & Intercept & 5.15642 & 3.94884 & -1.306 & 0.283 \\
\hline & & Entrance surface & 0.03131 & 0.01146 & 2.732 & 0.072 \\
\hline \multirow[t]{12}{*}{ Norway lobster } & \multirow[t]{6}{*}{ CAI } & Intercept & 7.48996 & 1.77919 & 4.210 & $<0.001$ \\
\hline & & Pot Volume & -0.03251 & 0.02366 & -1.374 & 0.172 \\
\hline & & Intercept & 9.41602 & 1.78556 & 5.273 & $<0.001$ \\
\hline & & Mesh size & -0.11416 & 0.04701 & -2.428 & 0.017 \\
\hline & & Intercept & 6.56545 & 0.67791 & 9.685 & $<0.001$ \\
\hline & & Entrance surface & -0.02723 & 0.01217 & -2.238 & 0.027 \\
\hline & \multirow[t]{6}{*}{ CBI } & Intercept & 9.268267 & 1.060265 & 8.741 & $<0.001$ \\
\hline & & Pot Volume & -0.005189 & 0.013958 & -0.372 & 0.711 \\
\hline & & Intercept & 8.0284 & 2.3069 & 3.480 & $<0.001$ \\
\hline & & Mesh size & 0.0230 & 0.0608 & 0.378 & 0.706 \\
\hline & & Intercept & 6.35333 & 0.79323 & 8.009 & $<0.001$ \\
\hline & & Entrance surface & 0.04856 & 0.01457 & 3.332 & 0.001 \\
\hline \multirow[t]{8}{*}{ Common octopus } & \multirow[t]{4}{*}{ CAI } & Intercept & 1.85369 & 0.27625 & 6.710 & $<0.001$ \\
\hline & & Pot volume & 0.04127 & 0.01158 & 3.564 & 0.002 \\
\hline & & Intercept & 1.509127 & 0.573997 & 2.629 & 0.016 \\
\hline & & Entrance surface & 0.009493 & 0.004887 & 1.942 & 0.066 \\
\hline & \multirow[t]{4}{*}{ CBI } & Intercept & 8.547802 & 0.210538 & 40.6 & $<0.001$ \\
\hline & & Pot volume & 0.040982 & 0.008847 & 4.632 & $<0.001$ \\
\hline & & Intercept & 8.082064 & 0.445201 & 18.154 & $<0.001$ \\
\hline & & Entrance surface & 0.010542 & 0.003723 & 2.831 & 0.010 \\
\hline \multirow[t]{12}{*}{ Pandalid shrimps } & \multirow[t]{6}{*}{ CAI } & Intercept & 9.484657 & 0.403208 & 23.523 & $<0.001$ \\
\hline & & Pot Volume & 0.000770 & 0.002841 & 0.271 & 0.787 \\
\hline & & Intercept & 9.42791 & 0.35440 & 26.602 & $<0.001$ \\
\hline & & Mesh size & -0.05366 & 0.01716 & -3.127 & 0.002 \\
\hline & & Intercept & 12.547957 & 0.795035 & 15.783 & $<0.001$ \\
\hline & & Entrance surface & -0.02567 & 0.007333 & -3.501 & $<0.001$ \\
\hline & \multirow[t]{6}{*}{ CBI } & Intercept & 11.055733 & 0.225956 & 48.93 & $<0.001$ \\
\hline & & Pot volume & -0.00288 & 0.001714 & -1.68 & 0.097 \\
\hline & & Intercept & 9.43226 & 1.34677 & 7.004 & $<0.001$ \\
\hline & & Mesh size & 0.05106 & 0.06635 & 0.769 & 0.444 \\
\hline & & Intercept & 9.061715 & 0.895334 & 10.121 & $<0.001$ \\
\hline & & Entrance surface & 0.013419 & 0.008013 & 1.675 & 0.010 \\
\hline
\end{tabular}

$p$ values $<0.05$ are in bold 
Table 3 Results of Kruskal-Wallis test $\left(\chi^{2}\right)$ where CAI (N.ind $\left.\cdot \operatorname{pot}^{-1} \cdot \mathrm{yr}^{-1}\right)$ and CBI $\left(\mathrm{g} \cdot \mathrm{pot}^{-1} \cdot \mathrm{year}^{-1}\right)$ are response variables and pot shape, pot colour, entrance shape, entrance position and bait type are factorial covariates

\begin{tabular}{|c|c|c|c|c|c|c|c|c|c|}
\hline Species & $\begin{array}{l}\text { Catch } \\
\text { index }\end{array}$ & Factor & $\chi^{2}$ & $d f$ & $p$ value & Comparison & $\mathrm{Z}$ & $\begin{array}{l}P . \\
\text { adj }\end{array}$ & Conclusions \\
\hline \multirow[t]{6}{*}{ Spiny lobster } & \multirow[t]{3}{*}{ CAI } & Pot shape & 3.29 & 2 & 0.193 & & & & \\
\hline & & Pot colour & 1.4 & 3 & 0.706 & & & & \\
\hline & & $\begin{array}{l}\text { Entrance } \\
\text { position }\end{array}$ & 3.29 & 2 & 0.193 & & & & \\
\hline & \multirow[t]{3}{*}{ CBI } & Pot shape & 1.4087 & 1 & 0.235 & & & & \\
\hline & & Pot colour & 1.4087 & 1 & 0.235 & & & & \\
\hline & & $\begin{array}{l}\text { Entrance } \\
\text { position }\end{array}$ & 1.4087 & 1 & 0.235 & & & & \\
\hline \multirow[t]{10}{*}{ Norway lobster } & \multirow[t]{5}{*}{ CAI } & Pot shape & 1.3407 & 1 & 0.247 & & & & \\
\hline & & Pot colour & 5.8593 & 3 & 0.119 & & & & \\
\hline & & Entrance shape & 0.29103 & 1 & 0.59 & & & & \\
\hline & & $\begin{array}{l}\text { Entrance } \\
\text { position }\end{array}$ & 0 & 1 & 1 & & & & \\
\hline & & Bait type & 8.6071 & 1 & 0.003 & & & & MIX $>$ FH \\
\hline & \multirow[t]{5}{*}{ CBI } & Pot shape & 1.1443 & 2 & 0.564 & & & & \\
\hline & & Pot colour & 5.0333 & 3 & 0.169 & & & & \\
\hline & & Entrance shape & 2.17 & 2 & 0.338 & & & & \\
\hline & & $\begin{array}{l}\text { Entrance } \\
\text { position }\end{array}$ & 1.6124 & 2 & 0.446 & & & & \\
\hline & & Bait type & 7.0086 & 1 & 0.008 & & & & MIX $>$ FH \\
\hline \multirow{24}{*}{$\begin{array}{l}\text { Common } \\
\text { octopus }\end{array}$} & \multirow[t]{12}{*}{ CAI } & Pot shape & 6.5809 & 2 & 0.038 & Clay-Conical & -2.509969 & 0.037 & Clay $<$ Conical \\
\hline & & & & & & Clay-Cylindrical & -1.028849 & 0.303 & \\
\hline & & & & & & Conical-Cylindrical & 2.11565 & 0.052 & \\
\hline & & Pot colour & 6.5809 & 2 & $\mathbf{0 . 0 3 8}$ & Black-Brown & 1.5201217 & 0.193 & \\
\hline & & & & & & Black-Green & -1.5053113 & 0.159 & \\
\hline & & & & & & Brown-Green & -2.5099688 & 0.036 & Green $>$ Brown \\
\hline & & & & & & Black-Orange & 1.5867374 & 0.225 & \\
\hline & & & & & & Brown-Orange & -0.1195907 & 0.905 & \\
\hline & & & & & & Green-Orange & 2.6019053 & 0.056 & \\
\hline & & Entrance shape & 8.6015 & 1 & 0.003 & & & & $\mathrm{CF}>\mathrm{C}$ \\
\hline & & $\begin{array}{l}\text { Entrance } \\
\text { position }\end{array}$ & 0.057259 & 1 & 0.811 & & & & \\
\hline & & Bait type & 8.6015 & 1 & 0.003 & & & & Crabs $>$ No bait \\
\hline & \multirow[t]{12}{*}{ CBI } & Pot shape & 6.9917 & 2 & $\mathbf{0 . 0 3}$ & Clay-Conical & -2.575159 & 0.030 & Clay $<$ Conical \\
\hline & & & & & & Clay-Cylindrical & -1.009699 & 0.313 & \\
\hline & & & & & & Conical-Cylindrical & 2.211413 & 0.041 & Conical $>$ Cylindrical \\
\hline & & Pot colour & 12.783 & 3 & 0.005 & Black-Brown & 1.7897942 & 0.110 & \\
\hline & & & & & & Black-Green & -1.3369168 & 0.217 & \\
\hline & & & & & & Brown-Green & -2.5751592 & 0.030 & Green $>$ Brown \\
\hline & & & & & & Black-Orange & 2.4065382 & 0.032 & Black $>$ Orange \\
\hline & & & & & & Brown-Orange & 0.2987225 & 0.765 & \\
\hline & & & & & & Green-Orange & 3.0541849 & 0.014 & Green $>$ Orange \\
\hline & & Entrance shape & 7.5835 & 1 & 0.006 & & & & $\mathrm{CF}>\mathrm{C}$ \\
\hline & & $\begin{array}{l}\text { Entrance } \\
\text { position }\end{array}$ & 0 & 1 & 1 & & & & \\
\hline & & Bait type & 7.5835 & 1 & 0.006 & & & & Crabs $>$ No bait \\
\hline
\end{tabular}


Table 3 continued

\begin{tabular}{|c|c|c|c|c|c|c|c|c|c|}
\hline Species & $\begin{array}{l}\text { Catch } \\
\text { index }\end{array}$ & Factor & $\chi^{2}$ & $d f$ & $p$ value & Comparison & $\mathrm{Z}$ & $\begin{array}{l}\text { P. } \\
\text { adj }\end{array}$ & Conclusions \\
\hline \multirow{15}{*}{$\begin{array}{l}\text { Pandalid } \\
\text { shrimps }\end{array}$} & \multirow[t]{9}{*}{ CAI } & \multirow[t]{6}{*}{ Pot shape } & \multirow[t]{6}{*}{11.721} & \multirow[t]{6}{*}{3} & \multirow[t]{6}{*}{0.008} & Conical-Cylindrical & 3.1382794 & 0.010 & \multirow{6}{*}{$\begin{array}{l}\text { Conical }>\text { Cylindrical } \\
\text { Conical }>\text { Parallelepiped }\end{array}$} \\
\hline & & & & & & $\begin{array}{l}\text { Conical- } \\
\quad \text { Parallelepiped }\end{array}$ & 2.7388302 & 0.018 & \\
\hline & & & & & & $\begin{array}{l}\text { Cylindrical- } \\
\text { Parallelepiped }\end{array}$ & -0.5570054 & 0.578 & \\
\hline & & & & & & Conical-Spherical & 1.8641887 & 0.125 & \\
\hline & & & & & & Cylindrical-Spherical & 1.0470241 & 0.354 & \\
\hline & & & & & & $\begin{array}{l}\text { Parallelepiped- } \\
\text { Spherical }\end{array}$ & 1.1552264 & 0.372 & \\
\hline & & Pot colour & 4.1059 & 3 & 0.25 & & & & \\
\hline & & Entrance shape & 4.7953 & 1 & 0.029 & & & & $\mathrm{CF}>\mathrm{OF}$ \\
\hline & & $\begin{array}{l}\text { Entrance } \\
\text { position }\end{array}$ & 4.2794 & 3 & 0.233 & & & & \\
\hline & \multirow[t]{6}{*}{ CBI } & \multirow[t]{2}{*}{ Pot shape } & 3.6657 & 3 & 0.30 & & & & \\
\hline & & & 11.889 & 2 & 0.003 & Black-Green & -0.6280587 & 0.530 & \\
\hline & & \multirow[t]{2}{*}{ Pot colour } & & & & Black-White & 0.8665949 & 0.579 & \\
\hline & & & & & & Green-White & 3.3640477 & 0.002 & Green $>$ White \\
\hline & & Entrance shape & 1.1412 & 1 & 0.285 & & & & \\
\hline & & $\begin{array}{l}\text { Entrance } \\
\text { position }\end{array}$ & 6.6203 & 3 & 0.085 & & & & \\
\hline
\end{tabular}

The results of Dunn's post hoc test are represented by $\mathrm{Z}$ statistic and adjusted $p$ value (P.adj) and are reported under Conclusions $p$ values $<0.05$ are in bold

and 3/5 factorial covariates, pot shape, pot colour and entrance position, whereas no data were available to test entrance shape and bait type.

\section{Norway lobster}

Neither catch index was significantly related to pot volume. In contrast, catch efficiency declined significantly with greater mesh size, albeit only in terms of specimen number (Fig. 2; CAI, $p$ value $=0.017$ ), indicating that pots with a larger mesh size catch fewer Norway lobster individuals, but without significant weight loss. A larger entrance surface was associated with a significantly lower number of specimens caught (CAI, $p=0.027$ ) and a significantly greater catch weight (CBI, $p=0.001)$. Therefore, a larger entrance catches fewer but larger individuals (Fig. 2).

No significant effects were found between catch efficiency and 4/5 factorial covariates: pot shape, colour, entrance shape and entrance position. As regards bait type, a significant effect-found for both $\mathrm{CAI}(p=0.003)$ and CBI $(p=0.008)$-indicated that mixed bait catches more and larger specimens than fish bait.

\section{Common octopus}

A larger pot volume was associated with significantly greater CAI and CBI (both, $p<0.001$ ); therefore, larger pots catch more and larger individuals (Fig. 2). Information on mesh size was not available, since most pot models targeting this species lack netting. Larger entrance surfaces involved a significantly greater CBI $(p=0.010)$, meaning that larger openings catch larger specimens (Fig. 2).

Pot shape had a weak effect on catch efficiency (CAI, $p=0.038$; CBI, $p=0.030$ ). Dunn's post-hoc test disclosed that conical pots are more efficient than clay pots for both indices (CAI, $p=0.037$; CBI, $p=0.030$ ); moreover, conical pots were more efficient than cylindrical pots in catching larger individuals (CBI, $p=0.041$ ). Pot colour significantly affected catch efficiency (CAI, $p=0.038$; CBI, $p=0.005)$. Green pots catch more and larger specimens than brown (CAI, $p=0.036$ for; $\mathrm{CBI}$, 
Fig. 2 Graphs illustrating significant relationships between the numerical covariates (Volume, Mesh size, Entrance surfae) and the catch abundance index CAI (N.ind $\cdot$ pot $^{-1} \cdot$ year $^{-1}$ ) and/or the catch biomass index, CBI

(g.pot ${ }^{-1}$ year $^{-1}$ ), as highlighted by the generalized linear model (GLM) for the four species analysed $(\mathrm{OCC}=$ common octopus, NEP = Norway lobster, $\mathrm{XKX}=$ pandalid shrimps). Volume expressed in litres (L), Mesh size in millimetres $(\mathrm{mm})$ and Entrance surface in square centimetres $\left(\mathrm{cm}^{2}\right)$. Solid lines: GLM regression; dashed lines: $95 \%$ confidence intervals
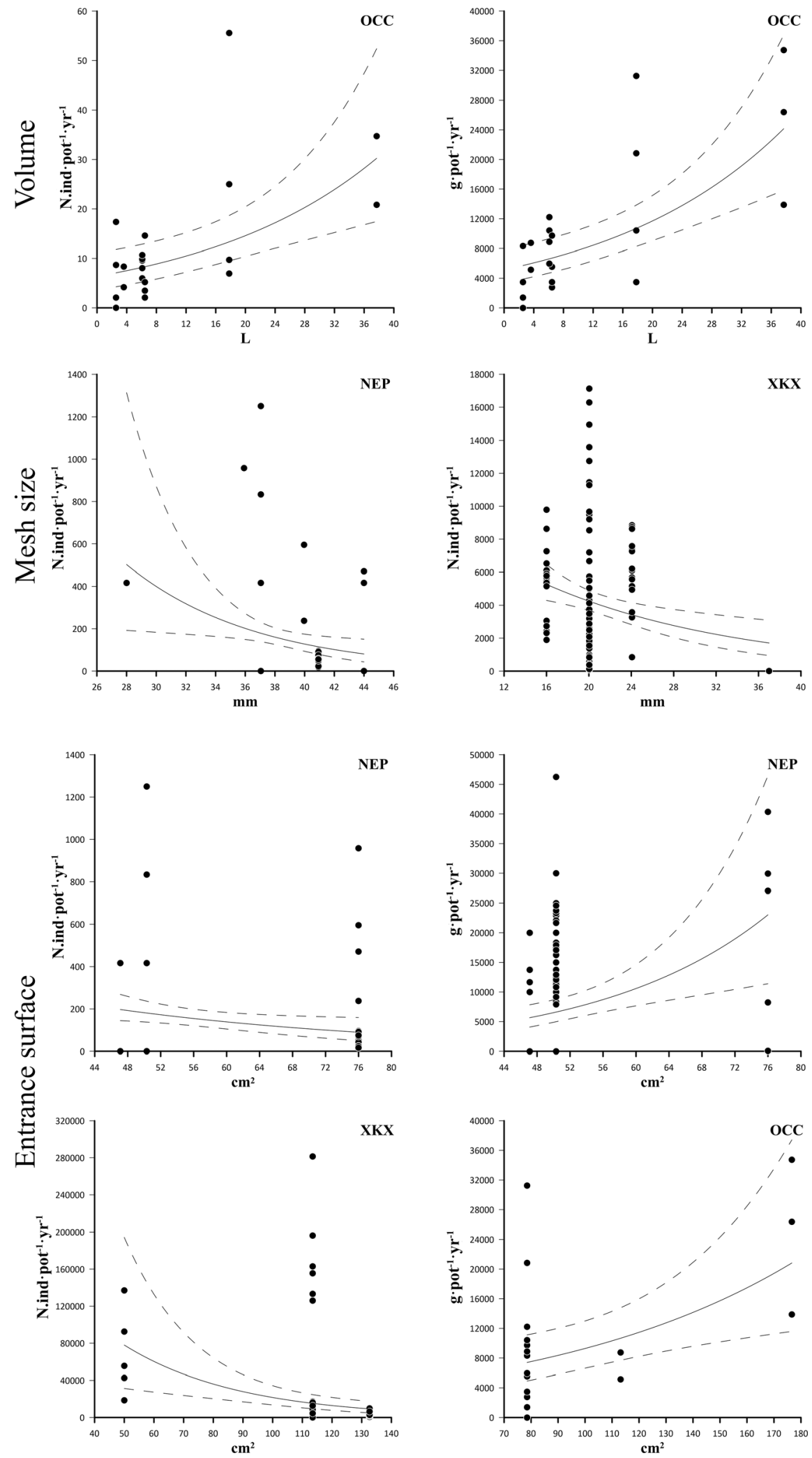
Table 4 SWOT analysis: factors and average scores

\begin{tabular}{|c|c|c|c|c|}
\hline Strengths & \multicolumn{2}{|l|}{$\mathrm{S}$} & Weaknesses & $\mathrm{S}$ \\
\hline Time- and labour-saving & \multicolumn{2}{|l|}{2.00} & Ghost fishing & 1.33 \\
\hline High quality of the catch & \multicolumn{2}{|l|}{2.00} & Early stage of research & 1.30 \\
\hline Minimization of habitat impacts & \multicolumn{2}{|l|}{1.88} & Specific target per pot design & 1.10 \\
\hline High size and species selectivity & \multicolumn{2}{|l|}{1.86} & Low catch of finfish species & 1.00 \\
\hline Discard reduction & \multicolumn{2}{|l|}{1.83} & Additional cost of bait & 0.86 \\
\hline Bycatch reduction & \multicolumn{2}{|l|}{1.77} & Storage on board & 0.50 \\
\hline Low energy use & \multicolumn{2}{|l|}{1.73} & & \\
\hline Low gear cost & \multicolumn{2}{|l|}{1.63} & & \\
\hline Ease of use & \multicolumn{2}{|l|}{1.43} & & \\
\hline Gear depredation avoidance & \multicolumn{2}{|l|}{1.40} & & \\
\hline \multicolumn{2}{|l|}{ Opportunities } & $S$ & Threats & $\mathrm{S}$ \\
\hline \multicolumn{2}{|l|}{ Responsible fisheries } & 2.00 & Spatial conflict with other coastal fisheries & 1.60 \\
\hline \multicolumn{2}{|l|}{ Fleet diversification (conversion) } & 1.92 & Catch performance competitiveness & 1.00 \\
\hline \multirow{3}{*}{\multicolumn{2}{|c|}{ Fishing possible in rough bottoms or marine protected areas }} & 1.91 & New regulations & 0.80 \\
\hline & & & Fisher appraisal & 0.67 \\
\hline & & & Economic competitiveness & 0.64 \\
\hline
\end{tabular}

$p=0.030)$ and orange $(\mathrm{CBI}, p$ value $=0.014)$ pots, whereas black pots catch larger individuals than orange pots $(\mathrm{CBI}, p$ value $=0.032)$. As regards entrance shape, a round opening with a funnel is more efficient than one without it, as demonstrated by both indices (CAI, $p=0.003$; CBI, $p=0.006$ ). Entrance position does not affect catch efficiency. Finally, pots baited with live crabs catch more (CAI, $p=0.003)$ and larger (CBI, $p=0.006)$ specimens than unbaited pots.

\section{Pandalid shrimps}

Neither catch index was significantly related to pot volume for these species. A greater mesh size yielded a negative effect in terms of the number of individuals caught (CAI, $p=0.002$ ), meaning that pots with a larger mesh size catch significantly fewer specimens (Fig. 2). Moreover, a greater entrance surface entailed a highly significant reduction in the number of individuals caught (CAI, $p<0.001$; Fig. 2).

Pot shape showed a significant effect only on CAI $(p=0.008)$. Dunn's post-hoc test disclosed that conical pots are more efficient than parallelepiped $(p=0.018)$ and cylindrical $(p=0.010)$ pots.

Pot colour has a significant effect only on CBI ( $p=p=0.003$ ), green being more efficient than white $(p=0.002)$. Entrance shape has a marginal effect on CAI $(p=0.029)$, since the round opening with a funnel is more efficient than the oval opening with a funnel. No significant effect was found for the entrance position. Information on bait type was not available.

\section{SWOT analysis: key factors}

Twenty papers investigating pots, 15 in the Mediterranean and 5 in other areas, were selected for this analysis (see Suppl. Mat. 3). Of the 24 key factors identified, 10 were Strengths, 6 were Weaknesses, 3 were Opportunities and 5 were Threats (Table 4 and Suppl. Mat. 3). A score $>1$ was found for all Strengths and Opportunities, for 4 Weaknesses and 2 Threats. The scenario sketched by the analysis confirmed that pots are a valuable alternative to traditional gears in the Mediterranean Sea, at least for some target species. The Strengths of pots include the fact that they are time- and labour-saving; the high quality of the catch; the reduction of habitat impacts; a high size and species selectivity; discard reduction; and bycatch reduction, whereas Opportunities include responsible fishing, fleet diversification (conversion) and a wider range of fishing areas. However, 
Weaknesses, i.e., ghost fishing, the current early stage of research and the narrow range of target species per pot design, and Threats, i.e., conflicts with other fisheries (sharing of fishing grounds) and competition with other gears are negative factors that should be addressed to enhance sustainability also at the socioeconomic level.

\section{Discussion}

Distribution of pot fisheries in the Mediterranean Sea

Our review of the literature highlighted that pots are used throughout the Mediterranean to target a large number of species. It also provided detailed information on existing pot fisheries and their evolution in the past few decades also compared to other traditional gears targeting the same species. In the Mediterranean, pots are currently used only in SSFs, whose vessels are less than 12 m long (Council Regulation 1198/2006).

The importance of pot fisheries varies in relation to the season and the geographical area. The season determines target species availability; for instance, on the Italian side of the Adriatic Sea cuttlefish is targeted by pots in spring and early summer, when females migrate to coastal areas in search of substrates on which to lay their eggs, and males follow them (Melli et al. 2014). Traditional pot fisheries play an important role in some countries. In North Africa and Spain the clay pots called gargoulettes, alcatrucez or cadups are employed since ancient times to catch common octopus (Lamboeuf 2001; Urbistondo 2001; Tsangridis et al. 2002; Chédia et al. 2010), whereas in Greece and Spain traditional round or square pots are used to catch Narwal shrimp, Plesionika narval (García-Rodriguez et al. 2000; Maravelias et al. 2018; Vasilakopoulos et al. 2019). Traditional pot fisheries targeting changeable nassa and cuttlefish are also widespread on the Italian side of the Adriatic Sea (Grati et al. 2010; Melli et al. 2014).

In other areas, the pots that used to be deployed to target other species have largely been replaced by more efficient gears; for instance, in the western Mediterranean the introduction of new materials and hauling techniques in the 1960s led to the adoption of trammel nets to catch spiny lobster, virtually superseding pots (Goñi et al. 2001, 2003). Pots now tend to be employed occasionally and in small number, especially to integrate the catches of other gears such as passive nets (Pretti 2019). Yet, in some areas pots are increasingly being used instead of other gears: they are deployed in Croatia to catch Norway lobster, when fishing closures stop bottom trawling (Brčić et al. 2017a), in the western Adriatic Sea to target mantis shrimp, which is traditionally caught with gill nets (Bon et al. 2006), and to catch common octopus in Italy and Greece (clay and PVC pots), where these fisheries are only a few decades old and are becoming an alternative métier (Lefkaditou et al. 2004; Sbrana et al. 2008; Pretti 2019).

Technical parameters affecting pot catch efficiency

Pot structure has evolved over time and differs from area to area. Pots are often still made by the fishers themselves (Viale et al. 2013); currently they are often made of synthetic materials, which are cheaper, sturdier and more durable than natural fibres and wood (Savo et al. 2013). In some cases, the high catch efficiency of a certain pot design has led to its diffusion to other areas of the Mediterranean. This is the case of the Spanish pot employed to catch pandalid shrimps. This semi-floating pot has a truncated-cone shape and rotates around an axis created by the clip and the float, which ensures that the round entrance always faces the current, catching pandalid shrimps that follow the odour plume (Guennegan 1990). It was originally developed in the western Mediterranean (GarcíaRodriguez et al. 2000) and the central-eastern Atlantic (Santana et al. 1997) and has proved highly effective in other areas of the Mediterranean (Guennegan 1990; Addis et al. 1998; Colloca 2002; Lelli 2006; Sartor et al. 2006; Possenti et al. 2007; Colloca and Lelli 2012).

Whereas a given pot design is usually developed to target one main species and a small number of accessory species (Pranovi et al. 2016; Grati et al. 2018), analysis of our dataset disclosed that a species is often targeted by more than one type of pot. In fact, pots can differ by several technical parameters, each of which potentially affects catch efficiency. Although the catch data were not reported in all the references reviewed, the statistical test results support some considerations on the catchability of the main species targeted by pots. 


\section{Spiny lobster}

The lack of significant relationships between pot parameters and catch efficiency, found in this study, is due to the low catch rates of spiny lobster reported in most papers. For instance, the catch of three types of pots in Tunisia was almost zero (Gaamor et al. 2004). A very low yield was described when four different designs were tested in Cabrera National Park (Spain), although the traditional plastic pot provided a significantly higher catch than a Japanese collapsible prototype (Amengual-Ramis et al. 2016). However, the Japanese pots caught numerous octopus; this may have deterred the spiny lobster, which use chemosensory signals to detect and avoid octopus (Gristina et al. 2011).

The highest yields of spiny lobster obtained with pots (12-23 individuals per 40 pots) have been described by Goñi et al. (2003), who tested commercial pots in Columbretes Islands (Spain). Goñi and Latrouite (2005) found a poor retention of small specimens $(<70 \mathrm{~mm}$ carapace length) compared with trammel nets and reported that large specimens (> $130 \mathrm{~mm}$ carapace length) were less likely to enter the pots, mainly due to morphological impediments, or were able to eat the bait without entering.

At variance with our results, Amengual-Ramis et al. (2016) reported that dark green plastic pots caught more specimens than white ones and suggested that the more limited contrast between their darker netting material and the background could make them more appealing hiding places. The authors also found that doubling the mesh size reduced the bycatch rate by four times.

Studies of sex-related vulnerability to pots have yielded no clear results (Miller 1990; Goñi et al. 2003). Moreover, lobster catchability may also be influenced by several factors besides pot features such as abundance, reproduction, moulting phase, phase of the lunar cycle and water temperature (Miller 1990; Goñi et al. 2001).

\section{Norway lobster}

The significant relationships of the catch indices with mesh size and entrance surface provide important information on the catchability of this species. As regards mesh size, the lower number of smaller individuals caught with the larger mesh sizes agrees with the laboratory experiments reported by Brčić et al. (2018b), who predicted that Nephrops with less than 28 and $32 \mathrm{~mm}$ of carapace length would escape from creels with 36 and $40 \mathrm{~mm}$ mesh size, respectively. The authors emphasized the role of mesh size in selecting only above the reference size ( $>20 \mathrm{~mm}$ of carapace length, as per Regulation EC 1967/2006), since the smaller specimens have sufficient time to seek an escape. Accordingly, Soldo et al. (1999) reported that pots caught no immature specimens regardless of mesh size (36, 40, $44 \mathrm{~mm})$. According to Brčić et al. (2018a), pots caught significantly larger specimens than trawl nets, since the average size at first maturity (L50) obtained for pots with $41 \mathrm{~mm}$ mesh size were $28.8 \%-65.9 \%$ larger than the L50 reported by bottom trawl selectivity studies with $40 \mathrm{~mm}$ square and $50 \mathrm{~mm}$ diamond codend mesh sizes, respectively.

The entrance surface seems to be size-selective, since a larger entrance allows smaller individuals to escape, whereas a smaller opening prevents the larger specimens, which have long pincers, from even entering the pot. Therefore, the lower CAI and higher CBI associated with a larger entrance resulted in catches of fewer but larger individuals.

The preference for mixed bait (pieces of fish and crab) over fish alone, disclosed by the analysis of our data, contrasts with the data reported by Bjordal (1979), who found no significant differences between the attractiveness of herring and mackerel, squid and trout pellet, and mackerel and trout pellet. Morello et al. (2009) described a high rate of bait scavenging (mostly by isopods) during experimental trials conducted in the Pomo Pit (central Adriatic Sea) and suggested halving the soak time. They also noted that, in shallower water, pots were crowded by swimming crabs that consumed all the bait and acted as deterrent to the entrance of Nephrops. For this reason, some Scottish creels have escape gaps to reduce the catch of crab species and indirectly increase the Norway lobster catch (Adey 2007). In fact, sea trials conducted in Norway (Bjordal 1986) have shown that smaller Nephrops specimens were easily scared away from the pots by individuals of other species or by larger conspecifics, which appeared to have a greater tolerance of disturbances. 


\section{Common octopus}

The significantly greater catch in terms of specimen number and weight by larger pots is in line with data reported outside the Mediterranean. The size selectivity curves obtained by testing pots differing in volume in the Gulf of Cádiz were bell-shaped and similar to the gill net curves (Sobrino et al. 2011). Consequently, only specimens of a certain size can be caught with a pot of a given volume, larger pots catching larger specimens and smaller ones catching smaller specimens. These findings agree with data reported in Korean waters by Kim et al. (2015), who observed that the smaller tube-shaped experimental pot caught significantly fewer and smaller long arm octopus, $O$. minor than the larger commercial net pot. Barry et al. (2010) reported that pot size was more important than entrance surface in the North Pacific fishery targeting giant octopus, Enteroctopus dofleini. Nonetheless, our findings that a larger entrance surface catches larger, but not more specimens may indicate that large specimens are deterred by small entrances.

The pot shape results highlighted a major difference between the simpler designs-such as clay pots and PVC tubes-and the more structurally complex models, like the Spanish pot, since the latter can be baited and bait always provides significantly higher yields (Sartor et al. 2008; Sbrana et al. 2008; Pretti 2019).

Our review demonstrated that darker colours (black, green) are more efficient than orange and brown. This finding agrees with the results of Borges et al. (2015), whose tank experiments highlighted a preference of this species for black over lighter colours, whereas Okamoto et al. (2001) found that $O$. vulgaris and $O$. aegina showed a preference for red, orange and black over yellow, green, blue, white and transparent shelters, regardless of the background colour.

\section{Pandalid shrimps}

The significantly lower number of pandalid shrimps caught with pots with larger entrance surfaces is probably due to an increased escape rate compared with pots with smaller entrances. Escape through the entrance has been described by Kalogirou et al. (2019) as a major factor influencing pot selectivity, together with escape through the meshes and the likelihood of encountering or entering a pot. Nevertheless, the entrance surface of the pots targeting pandalid shrimps is usually larger than that of pots targeting Nephrops (Table 1), suggesting that, once inside, pandalid shrimps are less capable of escaping than Norway lobster, despite their smaller size and absence of pincers.

In line with our results, Kalogirou et al. (2019) found that a larger mesh size reduced the retention probability of the smaller Narwal shrimp. Pots with $12 \times 12 \mathrm{~mm}$ mesh size showed the best performance as they avoided specimens less than $12 \mathrm{~mm}$ of carapace length, which is their L50 (Anastasopoulou et al. 2017).

The significantly better performance of green compared with white pots contrasts with the widespread custom of using white netting (Colloca and Lelli 2012), which according to Guennegan (1990) is the most effective colour for these species. Notably, Lelli (2006) found no difference between traditional white plastic netting and a green metal net with $10 \mathrm{~mm}$ mesh bars.

Guennegan (1990) reported that the type of bait (small dogfish, sardine etc.) seems to have a limited role in attracting shrimp, but that salting preserved its attractiveness and prevented scavenging by amphipods.

Main advantages and weaknesses of pots as alternative gears

The literature review on Mediterranean pot fisheries allowed identifying their main advantages and the limitations hampering their diffusion as an alternative to traditional gears.

Tsagarakis et al. (2014) found that pot fisheries have the lowest discards (1.6-9\%) together with demersal longlines (0-9\%), whereas the discards of gill nets may exceed $40 \%$ and those of bottom trawls reach $90 \%$. The high species and size selectivity of pots is especially interesting in the case of target species with a high commercial value, like Norway lobster in the Adriatic Sea (Brčić et al. 2017a). Several European pot fisheries target this species outside the Mediterranean (Ungfors et al. 2013); notably, they account for approximately $20 \%$ of all Nephrops landings in Sweden (Ziegler and Valentinsson 2008) and for $15 \%$ of landings in weight and over $26 \%$ of landings in value in Portugal, since these specimens are larger and in better condition than those caught 
with bottom trawls (Leocádio et al. 2012). In these areas, pots have been reported to be much more selective for Nephrops (Adey 2007) than trawls, which produce high discard rates and large amounts of bycatch, mainly fish (Monteiro et al. 2001).

Quite often, the specimens retrieved from pots are uninjured and command a higher price (Kopp et al. 2020). Accordingly, the immediate release of undersized or unwanted catch could entail an exemption from the LO, provided that high survival rates are scientifically demonstrated for these species (EU Regulation, 1380/2013). For example, the compulsory immediate release of females has proved to be an effective management measure in the Canadian pot fishery targeting the snow crab, Chionoecetes opilio (Nguyen et al. 2017).

In the Mediterranean, pots can reduce interactions between passive nets and cetaceans, especially the bottlenose dolphin, Tursiops truncatus, which tear the net and also reduce catch abundance and quality, hence profits (Bearzi 2002; Buscaino et al. 2009). Nevertheless, dolphin bycatch and injury, by the net or by fishers, can affect populations (Bearzi 2002; Díaz López 2006), as reported in several SSFs (Lauriano et al. 2009). In contrast, pots minimize the access to the catch by large predators such as dolphins, sea turtles and stingrays, since their small opening physically prevents them from entering the pot and stealing the bait or the catch. Notably, it also prevents their accidental entrapment. A greater diffusion of pots would therefore help reduce the bycatch of sensitive and protected species. For instance, the pots tested in the north-western Adriatic to catch cuttlefish and some fish species could provide a viable alternative to passive nets (Petetta et al. 2020b), at least in spring and summer, when high incidental catches of the loggerhead sea turtle Caretta caretta are recorded in coastal areas (Lucchetti et al. 2017c). A shift from passive nets to baited pots has been effective in reducing the damage done by the grey seal, Halichoerus grypus to the Baltic Sea gill net fishery for Atlantic cod, showing that pots can be a useful alternative gear at least during part of the year (Königson 2011; Bryhn et al. 2014; Königson et al. 2015).

Pots have also been demonstrated to minimize habitat impacts all over the world (Suuronen et al. 2012). A case study in the Bay of Biscay (Atlantic Ocean) has found a swept area less than $2 \mathrm{~m}^{2}$ during retrieval, which is negligible compared with other fishing gears (Kopp et al. 2020). Ziegler and Valentinsson (2008) have reported that the pot fishery targeting Norway lobster on the western Swedish coast entails a significantly lower seafloor pressure and energy use per kilogram of catch than bottom trawling. In the Mediterranean Sea, testing in the Ligurian Sea (Sala et al. 2013) of the floating pots used to catch cod in Scandinavia (Furevik et al. 2008) has shown that they have a much more limited seabed impact than other gears (e.g. seines and gill nets), especially in sensitive habitats such as Posidonia meadows.

In Mediterranean pot fisheries energy use is reasonably low, since the small vessels of SSFs commonly exploit fishing grounds at a limited distance from the home port (Grati et al. 2018). Pot fishery equipment costs are usually limited, since the materials employed are commonly fairly inexpensive (Colloca and Lelli 2012; Amengual-Ramis et al. 2016) and can sometimes be recycled. For instance, the PVC tubes which-due to their lower cost, greater resistance and easier handling on board-are increasingly replacing clay pots to catch common octopus, are mostly obtained from containers originally used for other purposes (Pretti 2019). Some designs are more expensive. A study by Petetta et al. (2020b) has found that about seven alternative pots are more expensive than $100 \mathrm{~m}$ of trammel net, which is traditionally used in the Adriatic Sea and covers the same fishing ground; however, whereas set nets usually last a single season and are then too damaged to be repaired, pots last up to 2 years, thus offsetting their higher initial cost. Passive nets are also time- and labour-intensive to manage on deck; in particular, whereas their discards are untangled manually (Szynaka et al. 2018), those of pots are extracted easily and quickly (Petetta et al. 2020b).

Baiting involves additional costs (AmengualRamis et al. 2016). For example, the bait placed in pots targeting Nephrops in European waters accounts for $5-10 \%$ of its first-hand sale price (Ungfors et al. 2013). Less expensive bait is being tested in some crab fisheries (Vazquez Archdale and Kawamura 2011; Araya-Schmidt et al. 2019), as is synthetic bait (Dellinger et al. 2016), which would be a momentous innovation and would reduce the pressure on bait fish stocks.

The narrow range of commercially important species targeted by each pot design, compared with other gears (Battaglia et al. 2010; Maynou et al. 2011), 
is probably the main factor hindering their wider diffusion. Pots have been reported to have a lower capture efficiency than trawls, seines and passive nets, especially for finfish species (Suuronen et al. 2012). However, we found no data regarding a specific pot fishery targeting finfish in the Mediterranean except for groupers and other fish (mainly sparids), which are traditionally caught with spherical and cylindrical pots in the eastern Mediterranean, e.g. Turkey (Çekiç et al. 2005; Genc et al. 2005, 2011; Turan et al. 2016; Mavruk et al. 2018) and Lebanon (Sacchi and Dimech 2011). In contrast, fish pots are frequently used outside the Mediterranean (Slack-Smith 2001) where, they are successfully deployed to target coral reef species (Thomsen et al. 2010; Suuronen et al. 2012). Another economically viable example is the pot fishery for Pacific cod, Gadus macrocephalus which has been established in the Gulf of Alaska and the Bering Sea (Thomsen et al. 2010). In the Mediterranean, large $(1800 \mathrm{~L})$ parallelepiped-shaped experimental pots have been tested in the Ligurian Sea (Italy) and in Heraklion Bay (Greece) to catch fish (Sala et al. 2013; Papadopoulou et al. 2015), but catches have been poor. More promising results have been obtained with collapsible Croatian pots, tested in the Adriatic Sea (Petetta et al. 2020b). Although they trapped fewer species than the trammel net, they caught a wider range of commercial species than the traditional pots used in the area by artisanal fishers, which target only cuttlefish (Fabi et al. 2001). Moreover, their collapsible design allows easy storage on board small Mediterranean artisanal boats, a key feature that overcomes a major limitation of traditional pots (Guennegan 1990; Petetta et al. 2020b).

Ghost fishing - the fact that gear that is lost or abandoned at sea continues to catch organisms-is a critical problem worldwide. It affects especially set nets, driftnets, fisheries employing drifting and anchored fish aggregating devices and bottom trawls (Gilman et al. 2021). In the Mediterranean, the gears involved more often seem to be passive nets, like gill nets (in Izmir Bay, Turkey; Ayaz et al. 2006), longlines and, less commonly, trawls (Ayaz et al. 2010). Studies assessing the impact of ghost fishing by lost pots in the Mediterranean are not numerous. In Iskerendum Bay (Turkey), losses-due chiefly to bad weather conditions, gear conflict, bottom structure hindrance and abandonment-have been estimated to be around $8 \%$ per fishing season (Özyurt et al. 2008).
In Sardinia, the majority of fishers stated that pots are frequently lost (Viale et al. 2013). The extent of ghost fishing seems to depend on pot shape, target species and the presence of bait. For instance, Erzini et al. (2008) have found that lost spherical fish pots involved higher mortality rates than octopus pots and that some species (i.e. common octopus) were better at escaping than others. In European Nephrops fisheries, the fishing potential of creels has been found to be low after bait consumption (Adey et al. 2008), since the target species is only attracted by the bait, not by dead target or non-target species in the creels, thus reducing the eventual ghost fishing. Moreover, creels also allow non-target species to escape relatively easily (Ungfors et al. 2013). Pots made from natural fibres or biodegradable panels instead of some meshes would facilitate escape (Bilkovic et al. 2012) and go some way towards mitigating ghost fishing impacts.

Finally, pot fisheries often interact negatively with other fisheries, especially those using active gears (e.g., bottom trawls and dredges), with considerable problems. Pot fisheries usually thrive in areas where trawl fisheries are banned or impractical. Area closures for active gears- as in the Loch Torridon (Scotland) Nephrops fishery, where the area has been completely closed to trawling (Macher and Talidec 2008) - is a notable example of good practice.

\section{Conclusions}

The present review offers some insights into Mediterranean pot fisheries and on the ability of pots to provide an alternative to the traditional gears used in this region. Some pot designs, like the Spanish pot targeting pandalid shrimps, the Scottish pots targeting Norway lobster and the Croatian collapsible pot targeting fish, which have recently been tested in different Mediterranean areas, have shown a good capture performance compared with gears targeting the same species, with the added benefit of lower discards, bycatch and seabed impacts. Consequently, their use could be extended to other areas of the basin.

Although commercial pot fisheries currently account for a small fraction of the fishing sector, the scenario outlined by this study shows that the scientific community is testing and encouraging the use of lowimpact gears such as pots. In fact, an increasing number of European research projects are promoting 
pots and their diffusion both in areas overexploited by other gears and in as yet unexploited areas, trying to stimulate fishing fleet conversion and increasingly attracting fishers' interest.

The wider diffusion of pot fisheries in the Mediterranean would be not a return to the past, or to a lower technological level, but rather a step towards a more sustainable future. Its limited environmental impact also involves that the product of pot fisheries could be certified with a quality mark, such as the Marine Stewardship Council sustainability label, which in 2003 was awarded to Scotland's Loch Torridon Nephrops creel fishery (Macher and Talidec 2008), further capturing fishers' engagement and promoting fishery sustainability.

Authors' contributions Andrea Petetta: contributed equally to this work with Massimo Virgili. He designed the review, analysed the data, prepared figures and/or tables, authored or reviewed drafts of the paper and approved the final draft. Massimo Virgili: contributed equally to this work with Andrea Petetta. He conceived and designed the review, prepared figures and/or tables, authored or reviewed drafts of the paper and approved the final draft. Stefano Guicciardi analysed the data, performed the statistical analysis, and approved the final draft. Alessandro Lucchetti conceived and designed the review and was the scientific responsible of the study, authored or reviewed drafts of the paper, and approved the final draft.

Funding This study was conducted with the contribution of the LIFE Financial Instrument of the European Community, Life Delfi Project - Dolphin Experience: Lowering Fishing Interactions (LIFE18NAT/IT/000942).

Availability of data and material The data are available under request of the authors.

Code availability R software and the packages used are open source.

\section{Declarations}

Conflict of interest The authors declare that they have no known competing financial interests or personal relationships that could have appeared to influence the work reported in this paper.

Open Access This article is licensed under a Creative Commons Attribution 4.0 International License, which permits use, sharing, adaptation, distribution and reproduction in any medium or format, as long as you give appropriate credit to the original author(s) and the source, provide a link to the Creative Commons licence, and indicate if changes were made. The images or other third party material in this article are included in the article's Creative Commons licence, unless indicated otherwise in a credit line to the material. If material is not included in the article's Creative Commons licence and your intended use is not permitted by statutory regulation or exceeds the permitted use, you will need to obtain permission directly from the copyright holder. To view a copy of this licence, visit http://creativecommons.org/licenses/by/4.0/.

\section{References}

Addis P, Campisi S, Cuccu D et al (1998) Mesobathyal experimental fishing with fixed gears. Biol Mar Mediterr 5:638-648

Adey JM (2007) Aspects of the sustainability of creel fishing for Norway lobster, Nephrops norvegicus (L.), on the west coast of Scotland. PhD Thesis. University of Glasgow, Scotland, $\mathrm{p} 472$

Adey JM, Smith IP, Atkinson RJA et al (2008) "Ghost fishing" of target and non-target species by Norway lobster Nephrops norvegicus creels. Mar Ecol Prog Ser 366:119-127. https://doi.org/10.3354/meps07520

Agresti A (2015) Foundations of linear and generalized linear models. Wiley, New Jersey

Amengual-Ramis JF, Vazquez-Archdale M, Canovas-Perez C, Morales-Nin B (2016) The artisanal fishery of the spiny lobster Palinurus elephas in Cabrera National Park, Spain: comparative study on traditional and modern traps with trammel nets. Fish Res 179:23-32

Anastasopoulou A, Makantasi P, Kapiris K et al (2017) Reproductive biology of Plesionika narval in the SE Aegean Sea (Eastern Mediterranean). Mediterr Mar Sci 18:454-467

Araya-Schmidt T, Olsen L, Rindahl L et al (2019) Alternative bait trials in the Barents Sea snow crab fishery. PeerJ. https://doi.org/10.7717/peerj.6874

Ayaz A, Acarli D, Altinagac U et al (2006) Ghost fishing by monofilament and multifilament gillnets in Izmir Bay, Turkey. Fish Res 79:267-271

Ayaz A, Ünal V, Acarli D, Altinagac U (2010) Fishing gear losses in the Gökova Special Environmental Protection Area (SEPA), eastern Mediterranean, Turkey. J Appl Ichthyol 26:416-419. https://doi.org/10.1111/j.1439-0426. 2009.01386.x

Ayaz A, Altinagac U, Ozekinci U et al (2011) Effect of twine thickness on selectivity of gillnets for bogue, Boops boops, in Turkish waters. Mediterr Mar Sci 12:358-368

Bahamon N, Sardà F, Suuronen P (2007) Selectivity of flexible size-sorting grid in Mediterranean multispecies trawl fishery. Fish Sci 73:1231-1240. https://doi.org/10.1111/j. 1444-2906.2007.01460.x

Barry PD, Tamone SL, Tallmon DA (2010) Evaluation of the capture efficiency and size selectivity of four pot types in the prospective fishery for North Pacific giant octopus (Enteroctopus dofleini). Fish Bull 108:39-44

Battaglia P, Romeo T, Consoli P et al (2010) Characterization of the artisanal fishery and its socio-economic aspects in the central Mediterranean Sea (Aeolian Islands, Italy). Fish Res 102:87-97. https://doi.org/10.1016/j.fishres.2009.10. 013 
Bearzi G (2002) Interactions between cetacean and fisheries in the Mediterranean Sea. In: Cetaceans of the Mediterranean and Black Seas: state of knowledge and conservation strategies. A report to the ACCOBAMS Secretariat, Monaco, pp 86-105

Bilkovic DM, Havens KJ, Stanhope DM, Angstadt KT (2012) Use of fully biodegradable panels to reduce derelict pot threats to marine fauna. Conserv Biol 26:957-966. https:// doi.org/10.1111/j.1523-1739.2012.01939.x

Bjordal A (1979) Factors effecting creel capture of Norway lobster (Nephrops norvegicus) and Northern shrimp (Pandalus borealis), investigations by fishery and baiting experiments (in Norwegian). Masters thesis. University of Bergen, p 97

Bjordal A (1986) The behaviour of Norway lobster towards baited creels and size selectivity of creels and trawl. FiskeridirSkr 18:131-137

Bon G, Feliziani L, Doz M et al (2006) Innovative methods of analysis of the trap fisheries for Squilla mantis and Gobius niger. Biol Mar Mediterr 13:809-813

Bonanomi S, Brčić J, Herrmann B et al (2020) Effect of a lateral square-mesh panel on the catch pattern and catch efficiency in a Mediterranean bottom trawl fishery. Mediterr Mar Sci 21:105-115

Borges TC, Calixto P, Sendao J (2015) The common octopus fishery in South Portugal: a new shelter-pot. Mediterránea Ser Estud Biológicos 130-154. https://doi.org/10.14198/ mdtrra2015.esp.07

Bradai MN, Saidi B, Enajjar S (2018) Overview on Mediterranean Shark's Fisheries: Impact on the Biodiversity. Mar Ecol Abiotic Interact 211-230. https://doi.org/10.5772/ intechopen.74923

Brčić J, Herrmann B, Mašanović M et al (2017a) Influence of soak time on catch performance of commercial creels targeting Norway lobster (Nephrops norvegicus) in the Mediterranean Sea. Aquat Living Resour 30:36. https:// doi.org/10.1051/alr/2017035

Brčić J, Herrmann B, Sala A (2017b) Can a square-mesh panel inserted in front of the cod end improve size and species selectivity in Mediterranean trawl fisheries? Can J Fish Aquat Sci 75:704-713. https://doi.org/10.1139/cjfas-20170123

Brčić J, Herrmann B, Mašanović M et al (2018a) CREELSELECT - a method for determining the optimal creel mesh: case study on Norway lobster (Nephrops norvegicus) fishery in the Mediterranean Sea. Fish Res 204:433-440. https://doi.org/10.1016/j.fishres.2018.03.020

Brčić J, Herrmann B, Mašanović M et al (2018b) Size selection of Nephrops norvegicus (L.) in commercial creel fishery in the Mediterranean Sea. Fish Res 200:25-32. https://doi. org/10.1016/j.fishres.2017.12.006

Bryhn AC, Königson SJ, Lunneryd SG, Bergenius MAJ (2014) Green lamps as visual stimuli affect the catch efficiency of floating cod (Gadus morhua) pots in the Baltic Sea. Fish Res 157:187-192. https://doi.org/10.1016/j.fishres.2014. 04.012

Buscaino G, Buffa G, Sarà G et al (2009) Pinger affects fish catch efficiency and damage to bottom gill nets related to bottlenose dolphins. Fish Sci 75:537-544. https://doi.org/ 10.1007/s12562-009-0059-3
Casale P (2011) Sea turtle by-catch in the Mediterranean. Fish Fish. https://doi.org/10.1111/j.1467-2979.2010.00394.x

Çekiç M, Dal T, Başusta N, Gökçe MA (2005) Comparison of two different types of basket trap on fish catches in iskenderun bay. Turk J Vet Anim Sci 29:743-749

Chédia J, Widien K, Amina B (2010) Role of sea surface temperature and rainfall in determining the stock and fishery of the common octopus (Octopus vulgaris, Mollusca, Cephalopoda) in Tunisia. Mar Ecol 31:431-438. https:// doi.org/10.1111/j.1439-0485.2009.00345.x

Coll M, Lotze HK, Romanuk TN (2008) Structural degradation in mediterranean sea food webs: testing ecological hypotheses using stochastic and mass-balance modelling. Ecosystems 11:939-960. https://doi.org/10.1007/s10021008-9171-y

Colloca F (2002) Life cycle of the deep-water pandalid shrimp Plesionika edwardsii (Decapoda, Caridea) in the central Mediterranean sea. J Crustac Biol 22:775-783. https://doi. org/10.1651/0278-0372(2002)022

Colloca F, Lelli S (2012) Report of the FAO EastMed support to the fishing trials carried out off the South Lebanese Coast. GCP/INT/041/EC - GRE - ITA/TD-14. Athens

Colloca F, Cardinale M, Maynou F et al (2013) Rebuilding Mediterranean fisheries: a new paradigm for ecological sustainability. Fish Fish 14:89-109. https://doi.org/10. 1111/j.1467-2979.2011.00453.x

Colloca F, Garofalo G, Bitetto I et al (2015) The seascape of demersal fish nursery areas in the North Mediterranean Sea, a first step towards the implementation of spatial planning for trawl fisheries. PLoS ONE 10:1-25. https:// doi.org/10.1371/journal.pone.0119590

Colloca F, Scarcella G, Libralato S (2017) Recent trends and impacts of fisheries exploitation on Mediterranean stocks and ecosystems. Front Mar Sci 4:244. https://doi.org/10. 3389/fmars.2017.00244

Dana BG (2012) SWOT analysis to improve quality management production. Procedia-Social Behav Sci 62:319-324

De Juan S, Thrush SF, Demestre M (2007) Functional changes as indicators of trawling disturbance on a benthic community located in a fishing ground (NW Mediterranean Sea). Mar Ecol Prog Ser 334:117-129. https://doi.org/10. 3354/meps334117

Dellinger A, Plotkin J, Duncan B et al (2016) A synthetic crustacean bait to stem forage fish depletion. Glob Ecol Conserv 7:238-244. https://doi.org/10.1016/j.gecco.2016. 07.001

Despoti S, Milisenda G, Ligas A et al (2020) Marine spatial closures as a supplementary tool to reduce discards in bottom trawl fisheries: Examples from southern European waters. Fish Res 232:105714. https://doi.org/10.1016/j. fishres.2020.105714

Díaz López B (2006) Interactions between Mediterranean bottlenose dolphins (Tursiops truncatus) and gillnets off Sardinia, Italy. ICES J Mar Sci 63:946-951. https://doi.org/ 10.1016/j.icesjms.2005.06.012

Dyson RG (2004) Strategic development and SWOT analysis at the University of Warwick. Eur J Oper Res 152:631-640

Erzini K, Bentes L, Coelho R et al (2008) Catches in ghostfishing octopus and fish traps in the northeastern Atlantic Ocean (Algarve, Portugal). Fish Bull 106:321-327 
European Regulation (EU) 1198/2006 (2006) Council regulation of 27 July 2006 on the European Fisheries Fund. Off J Eur Union 9:224-267

European Regulation (EU) 1380/2013 (2013) Regulation on the Common Fisheries Policy, amending Council Regulations (EC) No 1954/2003 and (EC) No 1224/2009 and repealing Council Regulations (EC) No 2371/2002 and (EC) No 639/2004 and Council Decision 2004/585/EC. Off Jour Eur Union L354:22-61

European Regulation (EU) 1967/2006 (2006) Council regulation concerning management measures for the sustainable exploitation of fishery resources in the Mediterranean Sea. Off J Eur Union L409

Fabi G, Grati F, Lucchetti A, Scarcella G (2001) Preliminary results on the catches of Sepia officinalis obtained with three set gears in the Central Adriatic Sea. Biol Mar Mediterr 8:660-664

Fabi G, Sbrana M, Biagi F et al (2002) Trammel net and gill net selectivity for Lithognathus mormyrus (L., 1758), Diplodus annularis (L., 1758) and Mullus barbatus (L., 1758) in the Adriatic and Ligurian seas. Fish Res 54:375-388. https:// doi.org/10.1016/S0165-7836(01)00270-3

FAO (2020) The State of Mediterranean and Black Sea Fisheries 2020. General Fisheries Commission for the Mediterranean, Rome

Farriols MT, Ordines F, Somerfield PJ et al (2017) Bottom trawl impacts on Mediterranean demersal fish diversity: not so obvious or are we too late? Cont Shelf Res 137:84-102. https://doi.org/10.1016/j.csr.2016.11.011

Farrugio H (2013) Current situation of small-scale fisheries in the Mediterranean and Black Sea: strategies and methodologies for an effective analysis of the sector. In: Report of the first regional symposium on sustainable small-scale fisheries in the Mediterranean and Black Sea, pp 27-30

Ferretti F, Myers RA, Serena F, Lotze HK (2008) Loss of large predatory sharks from the Mediterranean Sea. Conserv Biol 22:952-964

Furevik DM, Humborstad O, Jørgensen T, Løkkeborg S (2008) Floated fish pot eliminates bycatch of red king crab and maintains target catch of cod. Fish Res 92:23-27. https:// doi.org/10.1016/j.fishres.2007.12.017

Gaamor A, Rjeibi O, Bdioui M, et al (2004) Les Pêcheries de la langouste rouge Palinurus elephas de la Région Nord de la Tunisie: Engin de pêche, Eléments biologiques et gestion. Rapport final du projet BRAID (in French), p 16

García-Rodriguez M, Esteban A, Perez Gil JL (2000) Considerations on the biology of Pleisionika edwardis (Brandt, 1851) (Decapoda, Caridae, Pandalidae) from experimental trap catches in the Spanish western Mediterranean Sea. Sci Mar 64:369-379. https://doi.org/10.3989/scimar.2000. $64 n 4369$

Genc E, Genc MA, Genc E et al (2005) Seasonal variation and pathology associated with helminthes infecting two serranids (Teleostei) of Iskenderun Bay (Northeast Mediterranean), Turkey. Turk J Fish Aquat Sci 33:29-33

Genc E, Oral M, Erol C (2011) The evaluation of gnathiid (Crustacea: Isopoda: Gnathidae) parasitism in goldblotch grouper (Epinephelus costae Staindahner, 1878) in the northeastern Mediterranean Sea using the self-organizing map (SOM). Parasitol Res 108:1417-1424. https://doi.org/ 10.1007/s00436-010-2188-0
GFCM (2018) Working Group on Stock Assessment of Demersal Species (WGSAD) GFCM and FAO headquarters, Final report. Rome, Italy, 13-18 November 2017, p 79

Gilman E, Musyl M, Suuronen P et al (2021) Highest risk abandoned, lost and discarded fishing gear. Sci Rep 11:1-11. https://doi.org/10.1038/s41598-021-86123-3

Goñi R, Latrouite D (2005) Review of the biology, ecology and fisheries of Palinurus spp. species of European waters: Palinurus elephas (Fabricius, 1787) and Palinurus mauritanicus (Gruvel, 1911). Cah Biol Mar 46:127-142

Goñi R, Reñones O, Quetglas A (2001) Dynamics of a protected Western Mediterranean population of the European spiny lobster Palinurus elephas (Fabricius, 1787) assessed by trap surveys. Mar Freshw Res 52:1577-1587

Goñi R, Quetglas A, Reñones O (2003) Differential catchability of male and female European spiny lobster Palinurus elephas (Fabricius, 1787) in traps and trammelnets. Fish Res 65:295-307. https://doi.org/10.1016/j.fishres.2003.09.021

Grati F, Polidori P, Scarcella G, Fabi G (2010) Estimation of basket trap selectivity for changeable nassa (Nassarius mutabilis) in the Adriatic Sea. Fish Res 101:100-107. https://doi.org/10.1016/j.fishres.2009.09.012

Grati F, Bolognini L, Domenichetti F et al (2015) The effect of monofilament thickness on the catches of gillnets for common sole in the Mediterranean small-scale fishery. Fish Res 164:170-177. https://doi.org/10.1016/j.fishres. 2014.11.014

Grati F, Aladžuz A, Azzurro E et al (2018) Seasonal dynamics of small-scale fisheries in the Adriatic Sea. Mediterr Mar Sci 19:21-35. https://doi.org/10.12681/mms.2153

Gristina M, Sinopoli M, Fiorentino F et al (2011) Shelter selection of the spiny lobster Palinurus elephas under different levels of Octopus vulgaris predation threat. Mar Biol 158:1331-1337. https://doi.org/10.1007/s00227-0111652-4

Guennegan Y (1990) Diversification des techniques de pêche artisanales en corse - Pêche de la crevette Pandalide Plesionika edwardsii; technique, prospection, etude biologique, commercialisation. Ifremer Report (in French), p 111

Guijarro B, Ordines F, Massutí E (2017) Improving the ecological efficiency of the bottom trawl fishery in the Western Mediterranean: It's about time! Mar Policy 83:204-214. https://doi.org/10.1016/j.marpol.2017.06.007

ICES (2007) Report of the Study Group on the Development of Fish Pots for Commercial Fisheries and Survey Purposes (SGPOT). In: ICES CM 2007/FTC:02. Dublin, Ireland, p 17

ICES (2008) Report of the Study Group on the Development of Fish Pots for Commercial Fisheries and Survey Purposes (SGPOT). In: ICESCM2008/ FTC:01. Tórshavn, Faroe Islands, p 48

ICES (2009) Report of the Study Group on the Development of Fish Pots for Commercial Fisheries and Survey Purposes (SGPOT). In: ICES CM 2009/FTC:10. Ancona, Italy, p 13

ISSCFG (2016) International Standard Statistical Classification of Fishing Gear. Handb. Fish. Stat. 2

Jennings S, Stentiford GD, Leocadio AM et al (2016) Aquatic food security: insights into challenges and solutions from an analysis of interactions between fisheries, aquaculture, food safety, human health, fish and human welfare, 
economy and environment. Fish Fish 17:893-938. https:// doi.org/10.1111/faf.12152

Kalogirou S, Pihl L, Maravelias CD et al (2019) Shrimp trap selectivity in a Mediterranean small-scale-fishery. Fish Res 211:131-140. https://doi.org/10.1016/j.fishres.2018.11. 006

Karakulak FS, Erk H (2008) Gill net and trammel net selectivity in the northern Aegean Sea, Turkey. Sci Mar 72:527-540. https://doi.org/10.3989/scimar.2008.72n3527

Kim SH, Lee KH, Park SW, Lee DG (2015) Study on the fishing performance of an alternative tubular-type pot for the common octopus, octopus minor, in Korean coastal waters. Iran J Fish Sci 14:76-86

Königson S (2011) Seals and fisheries: a study of the conflict and some possible solutions (Thesis). University of Gothenburg, Sweden

Königson SJ, Fredriksson RE, Lunneryd S-G et al (2015) Cod pots in a Baltic fishery: Are they efficient and what affects their efficiency? ICES J Mar Sci 72:1545-1554

Kopp D, Coupeau Y, Vincent B et al (2020) The low impact of fish traps on the seabed makes it an eco-friendly fishing technique. PLoS ONE 15:1-13. https://doi.org/10.1371/ journal.pone.0237819

Kumova CA, Altınağaç U, Öztekin A et al (2015) Effect of hanging ratio on selectivity of gillnets for bogue (Boops boops, L. 1758). Turkish J Fish Aquat Sci 15:561-567

Lamboeuf M (2001) Artisanal fisheries in Libya: census of fishing vessels and inventory of artisanal fishery metiers. FAO-COPEMED Proj. p 44

Lauriano G, Caramanna L, Scarnó M, Andaloro F (2009) An overview of dolphin depredation in Italian artisanal fisheries. J Mar Biol Assoc United Kingdom 89:921-929. https://doi.org/10.1017/S0025315409000393

Lefkaditou E, Haralabous J, Sarikas D et al (2004) The cephalopods in the small-scale fishery in the eastern Thracian Sea (NE Mediterranean). In: ICES CM 2004/CC: 19. p 12

Lelli S (2006) Evaluation and development of two fishing métiers within the Tyre fishery sector: report on field survey carried out in June and July 2006. AID 7461/RC/LBN Final Rep. p 52

Leocádio AM, Whitmarsh D, Castro M (2012) Comparing trawl and creel fishing for Norway Lobster (Nephrops norvegicus): Biological and economic considerations. PLoS ONE 7:e39567. https://doi.org/10.1371/journal.pone.0039567

Link JS, Watson RA (2019) Global ecosystem overfishing: Clear delineation within real limits to production. Sci Adv 5:1-12. https://doi.org/10.1126/sciadv.aav0474

Lucchetti A, Sala A (2012) Impact and performance of Mediterranean fishing gear by side-scan sonar technology. Can J Fish Aquat Sci 69:1806-1816

Lucchetti A, Notti E, Sala A, Virgili M (2017a) Multipurpose use of side-scan sonar technology for fisheries science. Can J Fish Aquat Sci 75:1652-1662. https://doi.org/10.1139/ cjfas-2017-0359

Lucchetti A, Vasapollo C, Virgili M (2017b) An interviewbased approach to assess sea turtle bycatch in Italian waters. Peer J 5:e3151. https://doi.org/10.7717/peerj.3151

Lucchetti A, Vasapollo C, Virgili M (2017c) Sea turtles bycatch in the Adriatic Sea set net fisheries and possible hot-spot identification. Aquat Conserv Mar Freshw Ecosyst 27:1176-1185. https://doi.org/10.1002/aqc.2787
Lucchetti A, Bargione G, Petetta A et al (2019) Reducing sea turtle bycatch in the Mediterranean mixed demersal fisheries. Front Mar Sci 6:387. https://doi.org/10.3389/fmars. 2019.00387

Lucchetti A, Virgili M, Petetta A, Sartor P (2020) An overview of gill net and trammel net size selectivity in the Mediterranean Sea. Fish Res 230:105677. https://doi.org/ 10.1016/j.fishres.2020.105677

Macher C, Talidec C (2008) From trawl to pots: a bio-economic analysis of gear change. In: IIFET. Nha Trang, Vietnam, p 17

Maravelias CD, Vasilakopoulos P, Kalogirou S (2018) Participatory management in a high value small-scale fishery in the mediterranean sea. ICES J Mar Sci 75:2097-2106. https://doi.org/10.1093/icesjms/fsy119

Martínez-Baños P, Maynou F (2018) Reducing discards in trammel net fisheries with simple modifications based on a guarding net and artificial light: Contributing to marine biodiversity conservation. Sci Mar 82:9-18. https://doi. org/10.3989/scimar.04710.03A

Mavruk S, Saygu İ, Bengil F et al (2018) Grouper fishery in the Northeastern Mediterranean: an assessment based on interviews on resource users. Mar Policy 87:141-148. https://doi.org/10.1016/j.marpol.2017.10.018

Maynou F, Recasens L, Lombarte A (2011) Fishing tactics dynamics of a Mediterranean small-scale coastal fishery. Aquat Living Resour 24:149-159

Melli V, Riginella E, Nalon M, Mazzoldi C (2014) From Trap to Nursery. Mitigating the Impact of an Artisanal Fishery on Cuttlefish Offspring. PLoS One 9:e90542. https://doi.org/ 10.1371/journal.pone.0090542

Miller RJ (1990) Effectiveness of crab and lobster traps. Can J Fish Aquat Sci 47:1228-1251

Monteiro P, Araújo A, Erzini K, Castro M (2001) Discards of the Algarve (southern Portugal) crustacean trawl fishery. Hydrobiologia 449:267-277

Morello EB, Antolini B, Gramitto ME et al (2009) The fishery for Nephrops norvegicus (Linnaeus, 1758) in the central Adriatic Sea (Italy): Preliminary observations comparing bottom trawl and baited creels. Fish Res 95:325-331. https://doi.org/10.1016/j.fishres.2008.10.002

Nguyen KQ, Winger PD, Morris C, Grant SM (2017) Artificial lights improve the catchability of snow crab (Chionoecetes opilio) traps. Aquac Fish 2:124-133. https://doi.org/10. 1016/j.aaf.2017.05.001

Notarbartolo di Sciara G (2016) Marine mammals in the Mediterranean Sea: an overview, 1st edn. Elsevier Ltd.

Notti E, Brčić J, De Carlo F et al (2016) Assessment of the relative catch performance of a surrounding net without the purse line as an alternative to a traditional boat seine in small-scale fisheries. Mar Coast Fish 8:81-91. https://doi. org/10.1080/19425120.2015.1095827

Okamoto M, Anraku K, Kawamura G, Tanaka Y (2001) Selectivity of color of shelter by Octopus vulgaris and $O$. aegina under different background colors. Bull Japanese Soc Sci Fish 67:672-677

Olsen L (2014) Baited pots as an alternative fishing gear in the Norwegian fishery for Atlantic cod (Gadus morhua). Master Thesis. The Artic University of Norway, p 109 
Özyurt C, Akamca E, Kiyağa V, Taşlıel A (2008) The rates and reasons of pots, lost during a fishing season in Iskenderun Bay. J Fish Aquat Sci 25:147-151

Palanques A, Puig P, Guillén J et al (2014) Effects of bottom trawling on the Ebro continental shelf sedimentary system (NW Mediterranean). Cont Shelf Res 72:83-98. https:// doi.org/10.1016/j.csr.2013.10.008

Papadopoulou K, Smith CJ, Apostolidis C, Karachle PK (2015) Fish pot trials in the Aegean Sea: first experimental results. In: 11th Panhellenic Symposium on Oceanography and Fisheries. Mytilene, Lesvos Island, Greece, pp 157-160

Petetta A, Herrmann B, Virgili M, et al (2020a) Estimating selectivity of experimental diamond (T0) and turned mesh (T90) codends in multi-species Mediterranean bottom trawl. Mediterr Mar Sci 21:545-557. https://doi.org/10. 12681/mms.22789

Petetta A, Vasapollo C, Virgili M et al (2020b) Pots vs trammel nets: A catch comparison study in a Mediterranean smallscale fishery. PeerJ 8:e9287. https://doi.org/10.7717/peerj. 9287

Pol MV, He P, Winger PD (2010) Proceedings of the International Technical Workshop on Gadoid Capture by Pots (GACAPOT). Massachusetts Div Mar Fish Tech Rep 40:107

Possenti E, Sartor P, De Ranieri S (2007) Reproductive biology of females of Plesionika edwardsii (Brandt, 1851) (Crustacea, Decapoda, Pandalidae) in the northern Tyrrhenian Sea (Western Mediterranean). Atti Della Soc Toscana Di Sci Nat Mem Ser B 114:91-98

Pranovi F, Colla S, Valeri P, Anelli Monti M (2016) Present and future status of artisanal fisheries in the Adriatic Sea (western Mediterranean Sea). Ocean Coast Manag 122:49-56. https://doi.org/10.1016/j.ocecoaman.2016.01. 004

Pretti C (2019) Caratterizzazione e impatto della pesca del polpo comune, Octopus vulgaris, con attrezzi assimilabili a "barattoli", nella fascia costiera del mar tirreno settentrionale e centrale. (in Italian). Mipaaft Final Rep. p 41

Pusch C (2011) Cod traps as an ecological sound fishing gear in German waters. In: International marine Mammal-Gillnet Bycatch Workshop. BN Federal Agency For Nature Conservation, $\mathrm{p} 22$

Russo T, Parisi A, Garofalo G et al (2014) SMART: a spatially explicit bio-economic model for assessing and managing demersal fisheries, with an application to italian trawlers in the strait of sicily. PLoS ONE 9:e86222. https://doi.org/10. 1371/journal.pone.0086222

Sacchi J, Dimech M (2011) Report of the FAO EastMed assessment of the fishing gears in Lebanon. FAO-EastMed Proj. GCP/INT/041/EC - GRE - ITA. 47

Sala A, Lucchetti A (2011) Effect of mesh size and codend circumference on selectivity in the Mediterranean demersal trawl fisheries. Fish Res 110:252-258. https://doi.org/ 10.1016/J.FISHRES.2011.04.012

Sala A, Lucchetti A, Buglioni G (2007) The influence of twine thickness on the size selectivity of polyamide codends in a Mediterranean bottom trawl. Fish Res 83:192-203. https:// doi.org/10.1016/j.fishres.2006.09.013

Sala A, Virgili M, De Carlo F et al (2013) Sviluppo di attrezzi alternativi alla sciabica da natante nelle marinerie della Regione Liguria. Programma Operativo di Cooperazione
Trasfrontaliera "Italia-Francia Marittimo 2007-2013”, Progetto Comunitario MARTE+. Report finale (in Italian), p 67

Santana JI, González JA, Lozano IJ, Tuset VM (1997) Life history of plesionika edwardsi (crustacea, decapoda, Pandalidae) around the Canary Islands, Eastern central Atlantic. African J Mar Sci 18:39-48

Sartor P, Rossetti I, Vannucci A, Viva C (2006) Experimental traps targeting the striped soldier shrimp Plesionika edwardsii (Brandt, 1851) in the Northern Tyrrhenian Sea. Biol Mar Mediterr 13:288-289

Sartor P, De Ranieri S, Sbrana M et al (2008) Sperimentazione di differenti tipologie di nasse per la pesca del polpo, $O c$ topus vulgaris, Cuvier, 1797, e valutazione delle possibilità di impiego di questi attrezzi lungo il litorale livornese (in Italian). Rapp. Final. Regolam. U.E. N. 1263/99 - SFOP. p 65

Sartor P, Li Veli D, De Carlo F, et al (2018) Reducing unwanted catches of trammel nets: Experimental results of the "guarding net" in the caramote prawn, penaeus kerathurus, small-scale fishery of the Ligurian sea (western mediterranean). Sci Mar 82:131-140. https://doi.org/10.3989/ scimar.04765.15B

Savo V, La Rocca A, Caneva G et al (2013) Plants used in artisanal fisheries on the Western Mediterranean coasts of Italy. J Ethnobiol Ethnomed 9:1-14. https://doi.org/10. 1186/1746-4269-9-9

Sbrana M, Rossetti I, Sartor P et al (2008) Testing different models of traps targeting Octopus vulgaris, cuvier 1797, in the Eastern Ligurian Sea. Biol Mar Mediterr 15:356-357

Slack-Smith RJ (2001) Fishing with traps and pots. FAO Man $1-62$

Sobrino I, Juarez A, Rey J et al (2011) Description of the clay pot fishery in the Gulf of Cadiz (SW Spain) for Octopus vulgaris: Selectivity and exploitation pattern. Fish Res 108:283-290. https://doi.org/10.1016/j.fishres.2010.12. 022

Soldo A, Cetinic P, Dulčić J (1999) Catch of Norway lobster, Nephrops norvegicus (L.), with lobster pot of different mesh size in the Eastern Adriatic. In: International symposium on responsible fisheries \& fishing techniques, pp 281-287

STECF (2020) The 2020 Annual Economic Report on the EU Fishing Fleet (STECF 20-06), EUR 28359 EN. Publications Office of the European Union, Luxembourg

Suuronen P, Chopin F, Glass C et al (2012) Low impact and fuel efficient fishing-Looking beyond the horizon. Fish Res 119-120:135-146. https://doi.org/10.1016/j.fishres.2011. 12.009

Szynaka MJ, Bentes L, Monteiro P, et al (2018) Reduction of by-catch and discards in the Algarve small-scale coastal fishery using a monofilament trammel net rigged with a guarding net. Sci Mar 82:121-129. https://doi.org/10.3989/ scimar.04734.16B

Team RC (2019) R: a language and environment for statistical computing. Vienna: The R Foundation for Statistical Computing. Available at http://www.R-project.org.

Thomsen B, Humborstad OB, Furevik DM (2010) Fish pots: fish behavior, capture processes, and conservation issues. Behav Mar Fishes Capt Process Conserv Chall. https://doi. org/10.1002/9780813810966.ch6 
Tokaç A, Özbilgin HÖ, Kaykaç H (2010) Selectivity of conventional and alternative codend design for five fish species in the Aegean Sea. J Appl Ichthyol 26:403-409. https://doi. org/10.1111/j.1439-0426.2009.01379.x

Tsagarakis K, Palialexis A, Vassilopoulou V (2014) Mediterranean fishery discards: review of the existing knowledge. ICES J Mar Sci 71:1219-1234. https://doi.org/10.4135/ 9781412953924.n678

Tsangridis A, Sánchez P, Ioannidou D (2002) Exploitation patterns of Octopus vulgaris in two Mediterranean areas. Sci Mar 66:59-68. https://doi.org/10.3989/scimar.2002. $66 n 159$

Turan C, Salihoğlu B, Özgür Özbek E, Öztürk B (2016) The Turkish Part of the Mediterranean Sea; marine biodiversity, fisheries, conservation and governance. Turkish Marine Research Foundation (TUDAV), Istanbul, Turkey. p 595

Ungfors A, Bell E, Johnson ML et al (2013) Nephrops Fisheries in European Waters, 1st edn. Elsevier, Amsterdam

Urbistondo JAA (2001) Inventario de la Pesca Artesanal en España Mediterránea (2000-2001). (in Spanish). FAOCOPEMED Proj. p 45

Vasapollo C, Virgili M, Petetta A et al (2019) Bottom trawl catch comparison in the Mediterranean Sea: flexible turtle excluder device (TED) vs traditional gear. PLoS ONE 14:e216023. https://doi.org/10.1371/journal.pone. 0216023

Vasilakopoulos P, Maravelias CD, Anastasopoulou A et al (2019) Premium small scale: the trap fishery for Plesionika narval (Decapoda, Pandalidae) in the eastern Mediterranean Sea. Hydrobiologia 826:279-290. https://doi.org/ 10.1007/s10750-018-3739-0

Vazquez Archdale M, Kawamura G (2011) Evaluation of artificial and natural baits for the pot fishery of the sand crab Ovalipes punctatus (De Haan, 1833). Fish Res 111:159-163. https://doi.org/10.1016/j.fishres.2011.07. 006

Viale I, Palomba A, Gerardi M (2013) Indagine conoscitiva sull'uso delle nasse in Sardegna. (in Italian). Laore Publ. p 28

Virgili M, Vasapollo C, Lucchetti A (2018) Can ultraviolet illumination reduce sea turtle bycatch in Mediterranean set net fisheries? Fish Res 199:1-7. https://doi.org/10.1016/j. fishres.2017.11.012

Witte RS, Witte JS (2017) Statistics, 11th edn. Wiley, New York Ziegler F, Valentinsson D (2008) Environmental life cycle assessment of Norway lobster (Nephrops norvegicus) caught along the Swedish west coast by creels and conventional trawls - LCA methodology with case study. Int J Life Cycle Assess 13:487-497. https://doi.org/10.1007/ s11367-008-0024-x

Publisher's Note Springer Nature remains neutral with regard to jurisdictional claims in published maps and institutional affiliations. 